\title{
An Investigation on Analytical Properties of Delayed Fractional Order HIV Model: A Case Study
}

\author{
Pitchaimani M., Saranya Devi A.* \\ Ramanujan Institute for Advanced Study in Mathematics, University of Madras, \\ Chennai-600005, India
}

\begin{abstract}
In this manuscript, we design a fractional order delay differential equation model for HIV transmission with the implementation of three distinct therapies for three different infectious stages. The novelty of the model lies in clubbing delay and fractional calculus with the introduction of three different therapies for HIV in a single model. We investigate the positivity of solutions, analyze the stability properties, followed by Hopf bifurcation analysis. To probe the parameters that expedite the spread of infection, uncertainty and sensitivity analysis were performed. The numerical review was carried out to substantiate our theoretical results. Our proposed model parameters have been calibrated to fit yearly data from Afghanistan, Australia, France, Italy, Netherlands and New Zealand.
\end{abstract}

Key words: fractional order system, HIV, Hopf bifurcation, stability, time delay.

\section{INTRODUCTION}

Acquired immunodeficiency syndrome (AIDS) is the leading infectious cause of adult death in the world. Many researchers have focused on modeling HIV, in order to understand its dynamics, as it is the best way to design medicine for HIV. An important advantage of using compartmental models is that the mathematical representation of biological processes allows for clarity and precision with respect to epidemiological hypotheses and aid to decision making [1]. It is well understood from the outset that the infectious disease is modeled using compartments in which it is presumed that the population has the same character in each compartment. From this it is possible to observe how the population passes through each compartment and why it passes using one compartment to another. As the infected individual progresses through different phases of infection before developing AIDS in a full swing, we frame the HIV phases into compartments and sketch out the characteristics of each phase, as shown in Fig. 1. Even though the progression of HIV infection is represented in stages, it is inevitable for a person to go from stage 1 (initial acute phase) to stage 3 (symptomatic phase). Several therapies are available which can prevent a patient from obtaining AIDS and alleviate the symptoms of HIV infection. For normal individuals, the $\mathrm{CD}^{+}{ }^{+}$cell count level is 800 to $1200 \mathrm{~mm}^{3}$, and for HIV individuals, 200 or less. HIV infection is almost invariably lethal within 5 to 10 years without drug treatment. People treated with HIV will live longer without the symptoms associated with HIV. Currently, 79 percentage of people with HIV are believed to know their status. Antiretrovial therapy (ART) was offered worldwide to 23.3 million people living with HIV in 2018. New HIV infections started falling by 37 percentage between 2000 and 2018, and HIV-related deaths fell by 45 percentage with ART rescuing 13.6 million lives. This achievement was the result of great efforts by national HIV programmes supported by civil society and international development partners [1].

Mathematical models have been applied to the characterizing of infectious diseases in the last

*mathsaran430@gmail.com 
few decades and HIV epidemiologies occupy a major portion of research in the arena. In 2009, Cai et al. [2] have introduced a simple model of HIV with two stages, namely asymptomatic, symptomatic stages with treatment. This work was followed by Hernandez-Vargas and Middleton [3], who provided a HIV model with three stages. Followed by this, in the year of 2013, Sharma and Samanta [4] have compared a deterministic and delay model of HIV with its phases. Currently, in 2017, Simpson and Gumel [ 5 ] have designed a HIV model that incorporates pre-exposure prophylaxis (PrEP). Recently, in 2020, Akrami and Atabaigi [6] have analyzed about Hopf and forward bifurcation of an integer and fractional-order SIR epidemic model, followed by this in the same year Wu et al. [7] have studied about the complex behavior analysis of a fractional-order land dynamical model with holling-II type land reclamation rate on time delay. In 2020, Shaikh et al. [] ] have designed a fractional order covid-19 model. Numerous models where discussed in the literature in different dimensions, which have been tabulated in the Table 1.

Table 1. Literature survey

\begin{tabular}{|c|c|c|}
\hline S. No. & Types of HIV Models & References \\
\hline 1. & Models with treatment & {$[\underline{2}, \underline{5]}$} \\
\hline 2. & Models with HIV stages & {$[\underline{4}, \underline{\underline{3}}]$} \\
\hline 3. & Age structured models & {$[\underline{9}, \underline{10}]$} \\
\hline 4. & Co-infection models & {$[\underline{11}, \underline{12}]$} \\
\hline 5. & Models with delay & {$[\underline{13}, \underline{14}, \underline{15}, \underline{16}, \underline{17}]$} \\
\hline 6. & Fractional order models & {$[\underline{24}, \underline{25}, \underline{18}, \underline{19}, \underline{20}, \underline{21}, \underline{22}]$} \\
\hline 7. & Fractional order delay models & {$[\underline{34}, \underline{\underline{35}}, \underline{\underline{36}}]$} \\
\hline
\end{tabular}

Motivated by the above literature, we formulate a five compartmental HIV epidemic model with three different types of treatment namely AntiRetroviral therapy (ART), Protease Inhibitor (PI) and Reverse Transcriptase Inhibitor (RTI) which add up a novelty to our study. To derive our model equations, we divide the total high-risk human population (denoted by $N(t)$ ) into five different classes, namely, susceptible to the disease, $S(t)$, infective population in acute phase, $I(t)$, infective population in asymptomatic phase, $K(t)$, infective population in symptomatic phase, $H(t)$ and full-blown AIDS group $A(t)$ at time $t$. The natural death rate $\mu$ is assumed to be same for all compartments. The complete dynamics of system (1) is represented by a flow chart in Fig. 1. The description of parameters used in system (1) is provided in Table 2. The proposed model takes the following form as follows: 


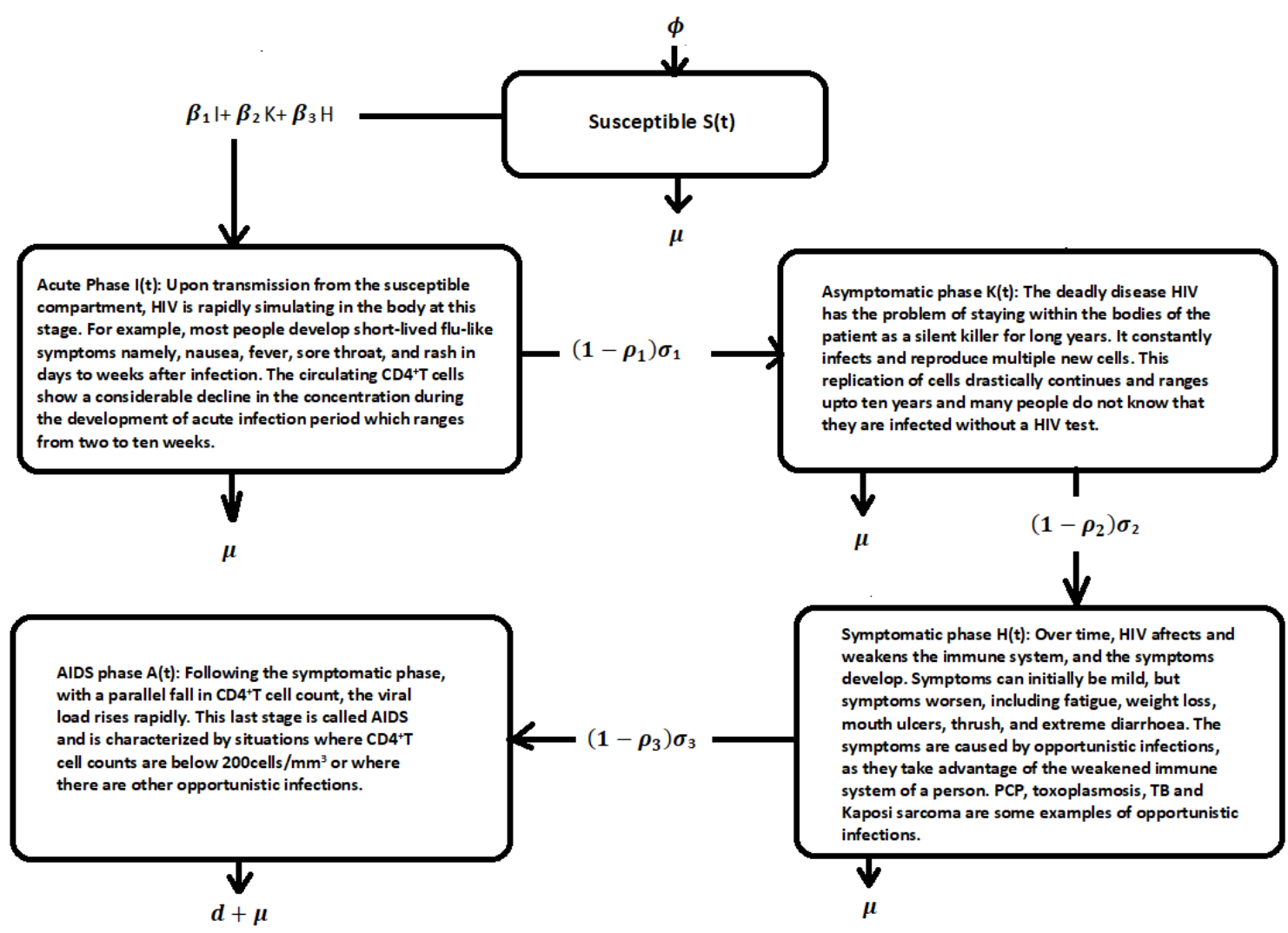

Fig. 1. Flowchart diagram for model (1)

$$
\begin{aligned}
& \frac{d S(t)}{d t}=\varphi-\beta_{1} I(t) S(t)-\beta_{2} K(t) S(t)-\beta_{3} H(t) S(t)-\mu S(t), \\
& \frac{d I(t)}{d t}=\beta_{1} I(t) S(t)+\beta_{2} K(t) S(t)+\beta_{3} H(t) S(t)-\left(1-\rho_{1}\right) \sigma_{1} I(t)-\mu I(t), \\
& \frac{d K(t)}{d t}=\left(1-\rho_{1}\right) \sigma_{1} I(t)-\left(1-\rho_{2}\right) \sigma_{2} K(t)-\mu K(t), \\
& \frac{d H(t)}{d t}=\left(1-\rho_{2}\right) \sigma_{2} K(t)-\left(1-\rho_{3}\right) \sigma_{3} H(t)-\mu H(t), \\
& \frac{d A(t)}{d t}=\left(1-\rho_{3}\right) \sigma_{3} H(t)-(d+\mu) A(t) .
\end{aligned}
$$

Fractional calculus has been 300 years old history, the development of fractional calculus theory is mainly focused on the pure mathematical field. The earliest more or less systematic studies seem to have been made in the $19^{t h}$ century by Liouville, Riemann, Leibniz, etc. Most differential systems used to describe physical phenomena are integer-order systems. With the development of fractional calculus, it has been found that the behavior of many systems can be described by using the fractional differential systems. It is worth mentioning that many physical phenomena having memory and genetic characteristics can be described by using the fractional differential systems. In fact, real world processes generally or most likely are fractional order systems. In recent years, the fractional dynamics have attracted the attention of many researchers due to their wide application in different fields, such as medicine, finance, hydrology, system biology and so on. Medicine and biology include rich sources for mathematical ideas. Mathematical modeling of diseases in biological studies is one of the most important application fields of fractional derivatives $[\underline{18}, \underline{19}, \underline{20}, \underline{21}, \underline{22}]$. 
In human societies, the process of evolution and control of the epidemic is associated with memory. The experience or knowledge of people about the spread of a disease in the past should affects their response. If people are aware of the past of a particular disease in their environment, they may use different precautionary measures, such as vaccinations. On the other hand, the fractional derivatives play an important role in describing the effects of memory in dynamic systems. As $\alpha$ limits to 1, the memory effects are reduced [리]

The fractional-order dynamical system can explain more realistic phenomena, which are neglected in the classical theory of dynamical system of integer order. Unlike integer-order models, fractional-order models have the potential to capture nonlocal relations in time. The fractional-order derivative gives more degree of freedom and consistent with reality of interactions because of its ability to give an exact expression of the nonlinear phenomena. The state of the integer order systems at each time does not depend on the previous history of the system: it is a memoryless process. It is well known that the behavior of the trajectory of a fractional differential operator is nonlocal and can be a useful way to include memory in a dynamical process. A dynamical process based on fractional order derivatives carries information about its present and past states. It is therefore reasonable to try to use a fractional compartmental system to observe the mechanism of the spread of diseases in epidemiological models. Biological models of fractional order have gained much attention in recent times which are more consistent for natural phenomena than integer-order systems that aid the characterization of dynamic systems such as memory property and fractional order system inherits some interesting properties from the integer order system $[\underline{24}, \underline{25}, \underline{26}, \underline{27}, \underline{28}, \underline{29}, \underline{30}$, $\underline{31}, \underline{32}, \underline{33}]$.

Most of the HIV models in literature have used the delay parameter to denote the time for uninfected population or cell to become infected population or cell. The inclusion of the delay parameter in the nonlinear system results in the rich dynamics of the system. Moreover, we know that delay impact disease spread in population. In the case of delay differential equation model, the shift in trajectories over time $t$ depends not only on $t$, but also on prior conditions. In the objective world, this sort of situation is plentiful. In our study, we replace the integer-order derivative into an arbitrary fractional order derivative $(0<\alpha \leq 1)$ and we incorporate the delay term $\tau$, where $\tau$ is the discrete time delay representing the time necessary for the susceptible humans to contact with the infectives $[\underline{35}, \underline{36}, \underline{37}, \underline{38}, \underline{39}, \underline{34}]$. Therefore, including these two forms of memories: a fractional order $(0<\alpha \leq 1)$ and discrete time delay $\tau$, in epidemic system (1) would certainly enrich the dynamics of the modified epidemic system (2). Many processes in nature possess memory and hereditary properties which cannot be modeled using ordinary derivative. There are two ways to include memory in the model. One called the "delay" and another is "fractional derivative". Due to its non local nature, fractional derivative is having a long memory. If these two things unified, the resulting model may be robust [ㅎ6].

We have system (2) is governed by fractional order delay differential equations of the form:

$$
\begin{aligned}
D_{t}^{\alpha} S(t)= & \varphi-\beta_{1} S(t) I(t-\tau)-\beta_{2} S(t) K(t-\tau)-\beta_{3} S(t) H(t-\tau)-\mu S(t), \\
D_{t}^{\alpha} I(t)= & \beta_{1} S(t) I(t-\tau)+\beta_{2} S(t) K(t-\tau)+\beta_{3} S(t) H(t-\tau) \\
& -\left(\delta_{1}+\mu\right) I(t), \\
D_{t}^{\alpha} K(t)= & \delta_{1} I(t)-\left(\delta_{2}+\mu\right) K(t), \\
D_{t}^{\alpha} H(t)= & \delta_{2} K(t)-\left(\delta_{3}+\mu\right) H(t), \\
D_{t}^{\alpha} A(t)= & \delta_{3} H(t)-(d+\mu) A(t),
\end{aligned}
$$

where $\left(1-\rho_{1}\right) \sigma_{1}=\delta_{1},\left(1-\rho_{2}\right) \sigma_{2}=\delta_{2}$ and $\left(1-\rho_{3}\right) \sigma_{3}=\delta_{3}$.

Fractional derivative of model (2) is in the sense of Caputo. Where $\alpha \in(0,1]$ is the order of 
the fractional derivative and $D_{t}^{\alpha}$ denotes $\frac{d^{\alpha}}{d t^{\alpha}}$. In the Caputo fractional operator standard initial conditions in terms of derivatives of integer order is involved. These initial conditions have clear physical interpretation. The Laplace transform of the Caputo Fractional derivative is a generalization of the Laplace transform of integer order derivative, where $n$ is replaced by $\alpha$. The same does not hold for the Riemann Liouville case. This property is an important advantage of the Caputo operator over the Riemann-Liouville operator [40]. The proposed fractional order HIV model (2) undergoes various analysis like local stability, bifurcation analysis and sensitivity analysis of the basic reproduction number $R_{0}$. We also present some numerical simulation for the proposed model and we fitted our model to yearly new cases of HIV infection for several countries like Afghanistan, Australia, France, Italy, Netherlands and New Zealand. The system (2), with the initial conditions

$$
\begin{array}{r}
S(\theta)=\psi_{1}(\theta), I(\theta)=\psi_{2}(\theta), K(\theta)=\psi_{3}(\theta), \\
H(\theta)=\psi_{4}(\theta), A(\theta)=\psi_{5}(\theta), \theta \in[-\tau, 0],
\end{array}
$$

where the initial function is denoted as $\psi=\left(\psi_{1}, \psi_{2}, \psi_{3}, \psi_{4}, \psi_{5}\right)^{T}$ belongs to $C:=$ $C\left([-\tau, 0], \mathbb{R}^{5}\right)$ of continuous functions mapping $[-\tau, 0]$ into $\mathbb{R}^{5}$. Since we deal with biological models, $\psi_{i} \geq 0, i=1,2,3,4,5$. Furthermore, we assume that

$$
S(t)>0, I(t) \geq 0, K(t) \geq 0, H(t) \geq 0, A(t) \geq 0, \text { for all } t>0 .
$$

The rest of this paper is systematized as follows. Section 2, concerns with the necessary preliminaries and the model analysis like local stability and bifurcation analysis of the system (2). In Section 3, sensitivity of the basic reproduction number $R_{0}$ have been performed. In Section 4, we present some numerical simulations and discussion for our system (2). In Section 5, we fitted our model to yearly new cases of HIV infection for Afghanistan, Australia, France, Italy, Netherlands and New Zealand. In Section 6, we give some concluding remarks. Finally, we discuss some future work in this direction in section 7 . The novelty of the model lies in clubbing delay and fractional calculus with the introduction of three different therapies for HIV in a single model. To the best of the authors knowledge, in such a complex model, this has not been addressed before in the literature.

\section{MODEL ANALYSIS}

In this section, we investigate some analytical properties of the nonlinear fractional order delay system $(2)$.

\subsection{PRELIMINARIES}

This subsection concerns with the necessary definitions, lemmas and theorems which will be used in the upcoming results.

Defin ition 1. [35] The Caputo fractional derivative of order $\alpha$ of a function $f(t) \in$ $C^{n}\left(\left[t_{1}, \infty\right), \mathbb{R}\right)$ is defined as

$$
D_{t}^{\alpha} f(t)=\frac{1}{\Gamma(n-\alpha)} \int_{t_{1}}^{t} \frac{f^{(n)}(\xi)}{(t-\xi)^{\alpha+1-n}} d \xi
$$

where $t_{1} \geq t, \Gamma($.$) is the Gamma function, and n$ is the positive integer such that $n-1<\alpha<n$. 
Table 2. Parameters description

\begin{tabular}{|c|c|c|c|}
\hline Parameter & Description & $\begin{array}{c}\text { Value } \\
\text { [assumed] }\end{array}$ & $\begin{array}{c}\text { Unit } \\
\text { [assumed] }\end{array}$ \\
\hline$\varphi$ & The recruitment rate & 500 & $d a y^{-1}$ \\
\hline$\beta_{1}$ & $\begin{array}{l}\text { Horizontal transmission rate coefficient of infection when } \\
\text { susceptibles contact with the infectives } \\
\text { in the first stage (acute phase) }\end{array}$ & 0.0000005 & $d a y^{-1}$ \\
\hline$\beta_{2}$ & $\begin{array}{l}\text { Horizontal transmission rate coefficient of infection when } \\
\text { susceptibles contact with the infectives } \\
\text { in the second stage (asymptomatic phase) }\end{array}$ & 0.0000006 & $d a y^{-1}$ \\
\hline$\beta_{3}$ & $\begin{array}{l}\text { Horizontal transmission rate coefficient of infection when } \\
\text { susceptibles contact with the infectives } \\
\text { in the third stage (symptomatic phase) }\end{array}$ & 0.0000002 & $d a y^{-1}$ \\
\hline$\mu$ & Natural death rate & 0.08 & $d a y^{-1}$ \\
\hline$\sigma_{1}$ & $\begin{array}{l}\text { Progression rate to second stage (asymptomatic phase) } \\
\text { from the first stage (acute phase) }\end{array}$ & 0.03 & $d a y^{-1}$ \\
\hline$\sigma_{2}$ & $\begin{array}{l}\text { Progression rate to third stage (symptomatic phase) } \\
\text { from the second stage (asymptomatic phase) }\end{array}$ & 0.05 & $d a y^{-1}$ \\
\hline$\sigma_{3}$ & $\begin{array}{l}\text { Progression rate to full-blown AIDS class from the } \\
\text { third stage (symptomatic phase) }\end{array}$ & 0.01 & $d a y^{-1}$ \\
\hline$\rho_{1}$ & Proportion of the efficacy of ART treatment & 0.65 & $d a y^{-1}$ \\
\hline$\rho_{2}$ & Proportion of the efficacy of PI treatment & 0.5 & $d a y^{-1}$ \\
\hline$\rho_{3}$ & Proportion of the efficacy of RTI treatment & 0.1 & $d a y^{-1}$ \\
\hline$d$ & Disease induced death rate of the full-blown AIDS group & 0.001 & $d a y^{-1}$ \\
\hline
\end{tabular}

When $0<\alpha<1$, one has

$$
D_{t}^{\alpha} f(t)=\frac{1}{\Gamma(1-\alpha)} \int_{t_{1}}^{t} \frac{f^{\prime}(\xi)}{(t-\xi)^{\alpha}} d \xi
$$


L e m m a 1. [35] Let $x(t)$ be a continuous function on $\left[t_{1},+\infty\right)$ and satisfy

$$
D_{t}^{\alpha} x(t) \leq-\lambda x(t)+\mu
$$

$x\left(t_{1}\right)=x_{t_{1}}$, where $0<\alpha \leq 1, \lambda, \mu \in \mathbb{R}$, and $\lambda \neq 0, t_{1} \leq 0$ is the initial time. Then

$$
x(t) \leq\left(x_{t_{1}}-\frac{\mu}{\lambda}\right) E_{\alpha}\left(-\lambda\left(t-t_{1}\right)^{\alpha}\right)+\frac{\mu}{\lambda},
$$

where $E_{\alpha}(\cdot)$ is the Mittag-Leffler function defined as

$$
E_{\alpha, \beta}(z)=\sum_{k=0}^{\infty} \frac{z_{k}}{\Gamma(k \alpha+\beta)},
$$

where, $\alpha>0, \beta>0$ and $z \in \mathbb{C}$. When $\beta=1$, one has $E_{\alpha}(z):=E_{\alpha, 1}(z)$. Furthermore, $E_{1,1}(z)=e^{z}$.

Th e or e m 1. [24] The following autonomous system

$$
\begin{gathered}
D_{t}^{\alpha} x(t)=A x, \\
x(0)=x_{0},
\end{gathered}
$$

with $0<\alpha \leq 1, x \in \mathbb{R}^{n}$ and $A \in \mathbb{R}^{n \times n}$ is asymptotically stable if $\left|\arg \left(\lambda_{j}\right)\right|>\frac{\alpha \pi}{2}$ is satisfied for all eigenvalues $\lambda_{j}$ of the matrix A. Also, this system is stable if and only if $\left|\arg \left(\lambda_{j}\right)\right| \geq \frac{\alpha \pi}{2}$ for all eigenvalues $\lambda_{j}$ of the matrix $A$ with those critical eigenvalues satisfying $\left|\arg \left(\lambda_{j}\right)\right|=\frac{\alpha \pi}{2}$ having geometric multiplicity of one. The geometric multiplicity of an eigenvalue $\lambda$ of the matrix $A$ is the dimension of the subspace of vectors $v$ for which $A v=\lambda v$.

Th e or e m 2. [24]. Consider the following commensurated fractional order system

$$
\begin{aligned}
D_{t}^{\alpha} x(t) & =f(x), \\
x(0) & =x_{0},
\end{aligned}
$$

with $0<\alpha \leq 1, x \in \mathbb{R}^{n}$ and $f: \mathbb{R}^{n} \rightarrow \mathbb{R}^{n}$ i.e., $f=\left(f_{1}, f_{2} \cdots f_{n}\right)^{T}$. The equilibrium points of the above system are calculated by solving the equation $f(x)=0$. These equilibrium points are locally asymptotically stable if all eigenvalues $\lambda_{j}$ of the Jacobian matrix $J=\frac{\partial f}{\partial x}$ evaluated at the equilibrium points satisfy $\left|\arg \left(\lambda_{j}\right)\right|>\frac{\alpha \pi}{2}$. The equilibrium points are said to be simply stable if $\left|\arg \left(\lambda_{j}\right)\right| \geq \frac{\alpha \pi}{2}$.

\subsection{WELL-POSEDNESS}

It is important to show the non-negativity and boundedness for the system (2) as they represent human populations. Positivity implies that the human population survives and the boundedness may be interpreted as a natural restriction to growth as a consequence of limited resources. Let us begin the analysis of the nonlinear system (2) by analyzing its positivity and boundedness. 


\subsubsection{Non-negativity and boundedness}

Since the problem taken up for analysis is biologically significant, we focus only on the solutions that are non-negative and bounded. Denote

$$
\mathbb{R}_{+}^{5}=\left\{x=\left(x_{1}, x_{2}, x_{3}, x_{4}, x_{5}\right) \mid x_{i} \geq 0, i \in\{1,2,3,4,5\}\right\} .
$$

Th e or e m 3. By the assumptions (3) and (4) the set

$\Omega=\left\{(S(t), I(t), K(t), H(t), A(t)) \in \mathbb{R}_{+}^{5} \mid S(t)+I(t)+K(t)+H(t)+A(t) \leq \frac{\varphi}{\mu}, t \geq 0\right\}$ is positively invariant with respect to the system (2).

$\mathrm{P} r \mathrm{r} \quad \mathrm{o} \mathrm{f}$. In order to prove that the system is always non-negative, we add all the compartments of the system (2), such that

$$
D_{t}^{\alpha} S(t)+D_{t}^{\alpha} I(t)+D_{t}^{\alpha} K(t)+D_{t}^{\alpha} H(t)+D_{t}^{\alpha} A(t) \leq \varphi-\mu(S(t)+I(t)+K(t)+H(t))-(d+\mu) A(t) .
$$

We know that all the parameters are positive, one can obtain: $D_{t}^{\alpha} N(t) \leq \varphi-\mu(N(t))$ where $N(t)=S(t)+I(t)+K(t)+H(t)+A(t)$.

By using Lemma 1 we get, $N(t) \leq\left(\frac{-\varphi}{\mu}+N(0)\right) E_{\alpha}\left(-\mu t^{\alpha}\right)+\frac{\varphi}{\mu}$.

Since $E_{\alpha}\left(-\mu t^{\alpha}\right) \geq 0$ when $N(0) \leq \frac{\varphi}{\mu}$, we have $N(t) \leq \frac{\varphi}{\mu}$. Therefore, the following set is positively invariant for the system (2),

$$
\Omega=\left\{(S(t), I(t), K(t), H(t), A(t)) \mid N(t) \leq \frac{\varphi}{\mu}\right\} .
$$

It is easy to see that $S(t), I(t), K(t), H(t)$ and $A(t)$ are bounded in a invariant set $\Omega$. This completes the proof.

Remark 1. It can be noted that the above methodology for proving positivity and boundedness for system (2) can be adapted to prove the same for the system (1) and we get the same invariant set $\Omega$.

The next subsection deals with the basic reproduction number of the system (2) which plays a vital role in disease transmission and the condition for existence of equilibrium points of the system (2).

\subsection{THE BASIC REPRODUCTION NUMBER AND EQUILIBRIUM POINTS}

The biological sense of the basic number of reproduction $\left(R_{0}\right)$ is specified as the average number of secondary infections caused during the infectious period by a single infectious individual. The basic reproduction number $\left(R_{0}\right)$ is used to calculate the transmission capacity of a disease, which also acts as a measure to determine if an infectious disease may or may not spread through a population. The disease dies out when $R_{0}<1$ and it spreads when $R_{0}>1$. The basic reproduction number for the nonlinear model (2) was obtained using the next-generation matrix method [41].

$$
\begin{gathered}
R_{0}=\frac{\varphi\left[\beta_{1}\left(\delta_{2}+\mu\right)\left(\delta_{3}+\mu\right)+\beta_{2} \delta_{1}\left(\delta_{3}+\mu\right)+\beta_{3} \delta_{1} \delta_{2}\right]}{\mu\left(\delta_{1}+\mu\right)\left(\delta_{2}+\mu\right)\left(\delta_{3}+\mu\right)} \\
R_{0}=R_{01}+R_{02}+R_{03}
\end{gathered}
$$


where

$$
R_{01}=\frac{\varphi \beta_{1}}{\mu\left(\delta_{1}+\mu\right)}, R_{02}=\frac{\varphi \beta_{2} \delta_{1}}{\mu\left(\delta_{1}+\mu\right)\left(\delta_{2}+\mu\right)}, R_{03}=\frac{\varphi \beta_{3} \delta_{1} \delta_{2}}{\mu\left(\delta_{1}+\mu\right)\left(\delta_{2}+\mu\right)\left(\delta_{3}+\mu\right)}
$$

The given nonlinear dynamical system (2) possesses two equilibrium points which are described below,

i) The disease free equilibrium point is $E_{0}\left(S_{0}, I_{0}, K_{0}, H_{0}, A_{0}\right)=E_{0}\left(\frac{\varphi}{\mu}, 0,0,0,0\right)$,

ii) The endemic equilibrium point is $E_{1}\left(S_{1}, I_{1}, K_{1}, H_{1}, A_{1}\right)$,

where

$$
\begin{array}{r}
S_{1}=\frac{\varphi}{\mu R_{0}}, I_{1}=\frac{\varphi\left(R_{0}-1\right)}{R_{0}\left(\delta_{1}+\mu\right)}, K_{1}=\frac{\varphi \delta_{1}\left(R_{0}-1\right)}{R_{0}\left(\delta_{1}+\mu\right)\left(\delta_{2}+\mu\right)}, \\
H_{1}=\frac{\varphi \delta_{1} \delta_{2}\left(R_{0}-1\right)}{R_{0}\left(\delta_{1}+\mu\right)\left(\delta_{2}+\mu\right)\left(\delta_{3}+\mu\right)}, A_{1}=\frac{\delta_{3} H_{1}}{(d+\mu)} .
\end{array}
$$

In the next subsection, we analyze the local stability of the equilibria.

\subsection{THE LOCAL STABILITY ANALYSIS}

In the following theorem we discuss the condition for the system (2) to be locally asymptotically stable at its disease free equilibrium point.

Th e or e m 4. The disease free equilibrium $E_{0}$ of the system (2) is locally asymptotically stable if $R_{0}<1$, provided that $\tau \geq 0$.

$\mathrm{P} \mathrm{r}$ o o $\mathrm{f}$. The Jacobian matrix and the corresponding characteristic equation of system (2) at the disease free equilibrium point $E_{0}$ is given by

$$
J\left(E_{0}\right)=\left[\begin{array}{ccccc}
-\mu & \frac{-\beta_{1} \varphi e^{-\lambda \tau}}{\mu} & \frac{-\beta_{2} \phi e^{-\lambda \tau}}{\mu} & \frac{-\beta_{3} \phi e^{-\lambda \tau}}{\mu} & 0 \\
0 & \frac{\beta_{1} \varphi e^{-\lambda \tau}}{\mu} & \frac{\beta_{2} \varphi e^{-\lambda \tau}}{\mu} & \frac{\beta_{3} \varphi e^{-\lambda \tau}}{\mu} & 0 \\
& -\left(\delta_{1}+\mu\right) & & & 0 \\
0 & \delta_{1} & -\left(\delta_{2}+\mu\right) & 0 & 0 \\
0 & 0 & \delta_{2} & -\left(\delta_{3}+\mu\right) & 0 \\
0 & 0 & 0 & \delta_{3} & -(d+\mu)
\end{array}\right]
$$

and

$$
\left(\lambda^{\alpha}+\mu\right)\left(\lambda^{\alpha}+d+\mu\right)\left(\lambda^{3 \alpha}+P_{1} \lambda^{2 \alpha}+P_{2} \lambda^{\alpha}+P_{3}-e^{-\lambda \tau}\left(P_{4} \lambda^{2 \alpha}+P_{5} \lambda^{\alpha}+P_{6}\right)\right)=0,
$$


where

$$
\begin{aligned}
& P_{1}=3 \mu+\delta_{1}+\delta_{2}+\delta_{3} \\
& P_{2}=3 \mu^{2}+2 \mu\left(\delta_{1}+\delta_{2}+\delta_{3}\right)+\delta_{1} \delta_{2}+\delta_{1} \delta_{3}+\delta_{2} \delta_{3}, \\
& P_{3}=\mu^{3}+\mu^{2}\left(\delta_{1}+\delta_{2}+\delta_{3}\right)+\mu\left(\delta_{1} \delta_{2}+\delta_{1} \delta_{3}+\delta_{2} \delta_{3}\right)+\delta_{1} \delta_{2} \delta_{3}, \\
& P_{4}=\frac{\varphi \beta_{1}}{\mu} \\
& P_{5}=2 \phi \beta_{1}+\frac{\varphi \delta_{1} \beta_{2}}{\mu}+\frac{\varphi \delta_{2} \beta_{1}}{\mu}+\frac{\varphi \beta_{1} \delta_{3}}{\mu}, \\
& P_{6}=\varphi \mu \beta_{1}+\varphi\left(\beta_{2} \delta_{1}+\beta_{1} \delta_{2}+\beta_{1} \delta_{3}\right)+\frac{\varphi}{\mu}\left(\beta_{3} \delta_{1} \delta_{2}+\beta_{2} \delta_{1} \delta_{3}+\beta_{1} \delta_{2} \delta_{3}\right) .
\end{aligned}
$$

Clearly, Eq. (7) has two negative real root $\lambda_{1}^{\alpha}=-\mu, \lambda_{2}^{\alpha}=-(d+\mu)$. The remaining roots are calculated by the following transcendental equation

$$
\lambda^{3 \alpha}+P_{1} \lambda^{2 \alpha}+P_{2} \lambda^{\alpha}+P_{3}-e^{-\lambda \tau}\left(P_{4} \lambda^{2 \alpha}+P_{5} \lambda^{\alpha}+P_{6}\right)=0 .
$$

Put $\tau=0$ in Eq. (7), i.e. for non delay case we get

$$
\lambda^{3 \alpha}+\lambda^{2 \alpha}\left(P_{1}-P_{4}\right)+\lambda^{\alpha}\left(P_{2}-P_{5}\right)+\left(P_{3}-P_{6}\right)=0,
$$

where $P_{1}-P_{4}, P_{2}-P_{5}$ and $P_{3}-P_{6}>0$ which can be written as

$$
\begin{aligned}
P_{1}-P_{4} & =2 \mu+\delta_{2}+\delta_{3}+\left(1-R_{01}\right)\left(\delta_{1}+\mu\right), \\
P_{2}-P_{5} & =\left(\delta_{1}+\mu\right)\left(\delta_{3}+\mu\right)\left(1-R_{01}\right)+\left(\delta_{1}+\mu\right)\left(\delta_{2}+\mu\right)\left(1-R_{02}\right) \\
& +\left(\delta_{2}+\mu\right)\left(\delta_{3}+\mu\right)\left(1-R_{03}\right), \\
P_{3}-P_{6} & =\left(1-R_{0}\right)\left(\delta_{1}+\mu\right)\left(\delta_{2}+\mu\right)\left(\delta_{3}+\mu\right) .
\end{aligned}
$$

Using the above expressions it is straightforward to show that the all the eigenvalues of Eq. (7) have no positive roots and satisfy the condition $\left|\arg \left(\lambda_{j}\right)\right|>\frac{\alpha \pi}{2}$, for $j=1,2, \cdots, 5$, if $R_{0}<1$, when $\tau=0$.

Let us look for the root distribution of Eq. (7), when $\tau>0$. If we begin by looking for the purely imaginary $\lambda=i \omega, \omega>0$ is a solution of Eq. (7), then

$$
-i \omega^{3 \alpha}-\omega^{2 \alpha} P_{1}+i \omega^{\alpha} P_{2}+P_{3}-e^{-(i \omega) \tau}\left(-\omega^{2 \alpha} P_{4}+i \omega^{\alpha} P_{5}+P_{6}\right)=0 .
$$

By seperating real and imaginary parts we have,

$$
\begin{gathered}
-\omega^{2 \alpha} P_{1}+P_{3}+\cos (\omega \tau) \omega^{2 \alpha} P_{4}=\cos (\omega \tau) P_{6}+\sin (\omega \tau) P_{5}, \\
-\omega^{3 \alpha}+\omega^{\alpha} P_{2}-\sin (\omega \tau) \omega^{\alpha} P_{4}=\cos (\omega \tau) \omega^{\alpha} P_{5}-\sin (\omega \tau) P_{6} .
\end{gathered}
$$

Squaring and adding the above equations, we get

$$
\omega^{6 \alpha}+\omega^{4 \alpha}\left(P_{1}^{2}-2 P_{2}-P_{4}^{2}\right)+\omega^{2 \alpha}\left(-2 P_{1} P_{3}+P_{2}^{2}-P_{5}^{2}+2 P_{4} P_{6}\right)+P_{3}^{2}-P_{6}^{2}=0,
$$

substitute $M_{3}=P_{1}^{2}-2 P_{2}-P_{4}^{2}, M_{4}=-2 P_{1} P_{3}+P_{2}^{2}-P_{5}^{2}+2 P_{4} P_{6}$ and $M_{5}=P_{3}^{2}-P_{6}^{2}$ in above equation, we obtain,

$$
\omega^{6 \alpha}+\omega^{4 \alpha} M_{3}+\omega^{2 \alpha} M_{4}+M_{5}=0 .
$$

Now all we need to prove is Eq. (10) has no positive root. It happens only when $M_{3}, M_{4}$ and 
$M_{5}$ is positive, where

$$
\begin{aligned}
M_{3} & =3 \mu^{2}+\delta_{1}^{2}+\delta_{2}^{2}+\delta_{3}^{2}+2 \mu\left(\delta_{1}+\delta_{2}+\delta_{3}\right)+\frac{2 \phi \beta_{1} \delta_{3}}{\mu}, \\
M_{4} & =\left(\delta_{1}+\mu\right)\left(\delta_{3}+\mu\right)\left(1-R_{01}\right)+\left(\delta_{1}+\mu\right)\left(\delta_{2}+\mu\right)\left(1-R_{02}\right) \\
& +\left(\delta_{2}+\mu\right)\left(\delta_{3}+\mu\right)\left(1-R_{03}\right)+3 \mu^{2}+2 \mu\left(\delta_{1}+\delta_{2}+\delta_{3}\right) \\
& +\delta_{1} \delta_{2}+\delta_{1} \delta_{3}+\delta_{2} \delta_{3}+2 \phi \beta_{1}+\frac{\varphi \delta_{1} \beta_{2}}{\mu}+\frac{\varphi \delta_{2} \beta_{1}}{\mu}+\frac{\varphi \beta_{1} \delta_{3}}{\mu} \\
& +2\left(\delta_{1}+\mu\right)\left(\delta_{2}+\mu\right)\left(\delta_{3}+\mu\right)\left(1-R_{0}\right), \\
M_{5} & =\left(1-R_{0}\right)\left(1+R_{0}\right)\left(\delta_{1}+\mu\right)^{2}\left(\delta_{2}+\mu\right)^{2}\left(\delta_{3}+\mu\right)^{2} .
\end{aligned}
$$

As $M_{3}, M_{4}$ and $M_{5}>0$ only when $R_{0}<1$. This shows that the Eq. (10) has no positive root if $R_{0}<1$. It is clear that all the eigenvalues of Eq. (7) satisfy the condition $\left|\arg \left(\lambda_{j}\right)\right|>\frac{\alpha \pi}{2}$, for $j=1,2, \cdots, 5$, if $R_{0}<1$. According to Theorem 2 , the disease free equilibrium $E_{0}$ of the system (2) is locally asymptotically stable.

The following theorem, clearly explains the condition under which the system (2) undergoes Hopf bifurcation.

\subsection{BIFURCATION ANALYSIS}

Th e or e m 5. The endemic equilibrium point $E_{1}$ of the system (2) is locally asymptotically stable for $\tau \in\left[0, \tau_{0}\right)$. Moreover, it undergoes a Hopf bifurcation at $E_{1}$, when $\tau=\tau_{j}(j=$ $0,1,2 \ldots)$.

$\mathrm{P} \mathrm{r}$ o $0 \mathrm{f}$. As the standard techniques in the theory of bifurcation analysis suggests, the Jacobian matrix of the system (2) at the endemic equilibrium point $E_{1}$ to identify the characteristic equation when $\tau>0$ as follows:

$$
J\left(E_{1}\right)=\left[\begin{array}{ccccc}
-(N+\mu) & -\beta_{1} S_{1} e^{-\lambda \tau} & -\beta_{2} S_{1} e^{-\lambda \tau} & -\beta_{3} S_{1} e^{-\lambda \tau} & 0 \\
N & \begin{array}{c}
\beta_{1} S_{1} e^{-\lambda \tau} \\
-\left(\delta_{1}+\mu\right)
\end{array} & \beta_{2} S_{1} e^{-\lambda \tau} & \beta_{3} S_{1} e^{-\lambda \tau} & 0 \\
& \delta_{1} & -\left(\delta_{2}+\mu\right) & 0 & 0 \\
0 & 0 & \delta_{2} & -\left(\delta_{3}+\mu\right) & 0 \\
0 & 0 & 0 & \delta_{3} & -(d+\mu)
\end{array}\right] \text {, }
$$

where $N=\beta_{1} I_{1}+\beta_{2} K_{1}+\beta_{3} H_{1}$.

The characteristic polynomial of the above matrix $J\left(E_{1}\right)$ can be written as

$$
X_{1}(\lambda)+X_{2}(\lambda) e^{-\lambda \tau}+X_{3} e^{-\lambda \tau}=0
$$

where

$X_{1}(\lambda)=\lambda^{5 \alpha}+q_{1} \lambda^{4 \alpha}+q_{2} \lambda^{3 \alpha}+q_{3} \lambda^{2 \alpha}+q_{4} \lambda^{\alpha}+q_{5}$,

$X_{2}(\lambda)=r_{1} \lambda^{4 \alpha}+r_{2} \lambda^{3 \alpha}+r_{3} \lambda^{2 \alpha}+r_{4} \lambda^{\alpha}$ and $X_{3}=r_{5}$.

The values of $q_{1}, q_{2}, q_{3}, q_{4}, q_{5}, r_{1}, r_{2}, r_{3}, r_{4}$ and $r_{5}$ can be seen in Appendix. When $\tau=0$, Eq. 
(11) becomes

$$
\lambda^{5 \alpha}+\left(q_{1}+r_{1}\right) \lambda^{4 \alpha}+\left(q_{2}+r_{2}\right) \lambda^{3 \alpha}+\left(q_{3}+r_{3}\right) \lambda^{2 \alpha}+\left(q_{4}+r_{4}\right) \lambda^{\alpha}+\left(q_{5}+r_{5}\right)=0 .
$$

The endemic equilibrium $E_{1}$ is locally asymptotically stable, if it satisfy the following Routh-Hurwitz conditions:

i) $q_{1}+r_{1}>0, q_{2}+r_{2}>0, q_{3}+r_{3}>0, q_{4}+r_{4}>0, q_{5}+r_{5}>0$,

ii) $\left(q_{1}+r_{1}\right)\left(q_{2}+r_{2}\right)\left(q_{3}+r_{3}\right)>\left(q_{3}+r_{3}\right)^{2}+\left(q_{1}+r_{1}\right)^{2}\left(q_{4}+r_{4}\right)$,

iii) $\left(\left(q_{1}+r_{1}\right)\left(q_{4}+r_{4}\right)-\left(q_{5}+r_{5}\right)\right)\left(\left(q_{1}+r_{1}\right)\left(q_{2}+r_{2}\right)\left(q_{3}+r_{3}\right)-\left(q_{3}+r_{3}\right)^{2}-\left(q_{1}+r_{1}\right)^{2}\left(q_{4}+r_{4}\right)\right)>$ $\left(q_{5}+r_{5}\right)\left(\left(q_{1}+r_{1}\right)\left(q_{2}+r_{2}\right)-\left(q_{3}+r_{3}\right)\right)^{2}+\left(q_{1}+r_{1}\right)\left(q_{5}+r_{5}\right)^{2}$.

From [39], assume $\lambda=i \omega=\omega\left(\cos \left(\frac{\pi}{2}\right)+i \sin \left(\frac{\pi}{2}\right)\right), \omega>0$ and by substituting $\lambda=i \omega$ in (11), we have

$$
\left(\epsilon_{1}+i \kappa_{1}\right)+\left(\epsilon_{2}+i \kappa_{2}\right) e^{-i \omega \tau}+X_{3} e^{-i \omega \tau}=0
$$

where

$$
\begin{aligned}
& \epsilon_{1}=\sum_{y=0}^{5} q_{5-y} \omega^{y \alpha} \cos \left(\frac{y \alpha \pi}{2}\right), \kappa_{1}=\sum_{y=0}^{5} q_{5-y} \omega^{y \alpha} \sin \left(\frac{y \alpha \pi}{2}\right), \\
& \epsilon_{2}=\sum_{y=1}^{4} r_{5-y} \omega^{y \alpha} \cos \left(\frac{y \alpha \pi}{2}\right), \kappa_{2}=\sum_{y=1}^{4} r_{5-y} \omega^{y \alpha} \sin \left(\frac{y \alpha \pi}{2}\right),
\end{aligned}
$$

where $q_{0}=r_{0}=1$.

By separating the real and imaginary parts, we have

$$
\begin{aligned}
& \epsilon_{1}+\epsilon_{2} \cos (\omega \tau)+\kappa_{2} \sin (\omega \tau)+X_{3} \cos (\omega \tau)=0, \\
& \kappa_{1}-\epsilon_{2} \sin (\omega \tau)+\kappa_{2} \cos (\omega \tau)-X_{3} \sin (\omega \tau)=0 .
\end{aligned}
$$

From Eq. (14), we obtain

$$
\cos (\omega \tau)=\frac{-\epsilon_{1}\left(\epsilon_{2}+X_{3}\right)+\kappa_{1} \kappa_{2}}{\left(\epsilon_{2}+X_{3}\right)^{2}+\kappa_{2}^{2}}, \sin (\omega \tau)=\frac{\kappa_{1}\left(\epsilon_{2}+X_{3}\right)-\epsilon_{1} \kappa_{2}}{\left(\epsilon_{2}+X_{3}\right)^{2}+\kappa_{2}^{2}} .
$$

Since $\cos ^{2} \omega \tau+\sin ^{2} \omega \tau=1$, we have

$$
\begin{gathered}
\omega^{10 \alpha}+h_{1} \omega^{9 \alpha}+h_{2} \omega^{8 \alpha}+h_{3} \omega^{7 \alpha}+h_{4} \omega^{6 \alpha}+h_{5} \omega^{5 \alpha} \\
+h_{6} \omega^{4 \alpha}+h_{7} \omega^{3 \alpha}+h_{8} \omega^{2 \alpha}+h_{9} \omega^{\alpha}+h_{10}=0 .
\end{gathered}
$$

The values of $h_{1}, h_{2}, h_{3}, h_{4}, h_{5}, h_{6}, h_{7}, h_{8}, h_{9}$ and $h_{10}$ can be seen in Appendix.

Let

$$
\begin{gathered}
h(\omega)=\omega^{10 \alpha}+h_{1} \omega^{9 \alpha}+h_{2} \omega^{8 \alpha}+h_{3} \omega^{7 \alpha}+h_{4} \omega^{6 \alpha}+h_{5} \omega^{5 \alpha}+h_{6} \omega^{4 \alpha}+h_{7} \omega^{3 \alpha}+h_{8} \omega^{2 \alpha}+h_{9} \omega^{\alpha}+h_{10}, \\
h(\omega)=0 .
\end{gathered}
$$

To locate the bifurcation point, we need to understand the nature of roots and their distribution in the phase plane. We analyze this from Eq. (11), in the form of two cases as follows:

Case (i). If $h_{i}>0, i=1,2, \cdots, 10$, then Eq. $\underline{(11)}$ has no root with zero real parts for all $\tau \geq 0$. 
From $h_{i}>0, i=1,2, \cdots, 10$, then $h(0)=h_{10}>0$ and $h^{\prime}(\omega)=10 \alpha \omega^{10 \alpha-1}+9 \alpha \omega^{9 \alpha-1}+$ $8 \alpha \omega^{8 \alpha-1}+7 \alpha \omega^{7 \alpha-1}+6 \alpha \omega^{6 \alpha-1}+5 \alpha \omega^{5 \alpha-1}+4 \alpha \omega^{4 \alpha-1}+3 \alpha \omega^{3 \alpha-1}+2 \alpha \omega^{2 \alpha-1}+\alpha \omega^{\alpha-1}$. Combining $\alpha>0, h_{i}>0, i=1,2, \cdots, 10$, then we claim that Eq. (15) has no real root and hence, Eq. (11) has no purely imaginary root. Note that $h_{10} \neq 0$, then $\bar{\lambda}=0$ is not a root of Eq. (11).

Case (ii). If $h_{i}<0, i=1,2, \cdots, 9, h_{10}>0$, Eq. (11) has a pair of purely imaginary roots $\pm i \omega_{0}$ when $\tau=\tau_{j}, j=0,1,2, \cdots$

where

$$
\tau_{j}=\frac{1}{\omega_{0}}\left[\arccos \left(\frac{-\epsilon_{1}\left(\epsilon_{2}+X_{3}\right)+\kappa_{1} \kappa_{2}}{\left(\epsilon_{2}+X_{3}\right)^{2}+\kappa_{2}^{2}}\right)+2 j \pi\right], j=0,1,2 \cdots,
$$

and $\omega_{0}$ is the unique positive root of (15).

From $h_{10}>0$ and $h(0)=h_{10}>0$. Then, by $\lim _{\omega \rightarrow \infty} h(w)=+\infty, h^{\prime}(\omega)>0$ for $\omega>0$, there exists a unique $\omega_{0}>0$ such that $h\left(\omega_{0}\right)=0$. Then, $\omega_{0}$ is a root of (15). From (14), we know that Eq. (11) with $\tau=\tau_{j},(j=0,1,2, \cdots)$ has a pair of purely imaginary roots $\pm i \omega_{0}$.

Let $\lambda \overline{(\tau)}=n(\tau)+i \omega(\tau)$ be the root of $\left(\underline{11)}\right.$, such that when $\tau=\tau_{j}$ satisfying $n\left(\tau_{j}\right)=0$ and $\omega\left(\tau_{j}\right)=\omega_{0}$. From Eq. (11), we have

$$
\begin{array}{r}
{\left[\frac{d \lambda}{d \tau}\right]^{-1}=\frac{X_{1}^{\prime}(\lambda)+X_{2}^{\prime}(\lambda) e^{-\lambda \tau}-\tau X_{2}(\lambda) e^{-\lambda \tau}-\tau X_{3} e^{-\lambda \tau}}{\lambda\left(X_{2}(\lambda) e^{-\lambda \tau}+X_{3} e^{-\lambda \tau}\right)},} \\
X_{1}^{\prime}(\lambda)+X_{2}^{\prime}(\lambda) e^{-\lambda \tau}-\tau X_{2}(\lambda) e^{-\lambda \tau}-\tau X_{3} e^{-\lambda \tau}=a_{1}+i a_{2}, \\
\lambda\left(X_{2}(\lambda) e^{-\lambda \tau}+X_{3} e^{-\lambda \tau}\right)=a_{3}+i a_{4} .
\end{array}
$$

Where

$$
\begin{aligned}
a_{1} & =\sum_{y=1}^{5} q_{5-y} \omega^{y \alpha-1} \cos \left(\frac{(y \alpha-1) \pi}{2}\right)+\sum_{y=1}^{4} r_{5-y} \omega^{y \alpha-1} \cos \left(\omega \tau-\frac{(y \alpha-1) \pi}{2}\right) \\
& -\sum_{y=1}^{4} r_{5-y} \tau \omega^{y \alpha} \cos \left(\omega \tau-\frac{y \alpha \pi}{2}\right)-\tau X_{3} \sin (\omega \tau), \\
a_{2}= & \sum_{y=1}^{5} q_{5-y} \omega^{y \alpha-1} \sin \left(\frac{(y \alpha-1) \pi}{2}\right)+\sum_{y=1}^{4} r_{5-y} \omega^{y \alpha-1} \sin \left(\omega \tau-\frac{(y \alpha-1) \pi}{2}\right) \\
& -\sum_{y=1}^{4} r_{5-y} \tau \omega^{y \alpha} \sin \left(\omega \tau-\frac{y \alpha \pi}{2}\right)-\tau X_{3} \cos (\omega \tau), \\
a_{3}= & \sum_{y=1}^{4} r_{5-y} \omega^{y \alpha+1} \cos \left(\omega \tau-\frac{(y \alpha+1) \pi}{2}\right)+\omega X_{3} \sin (\omega \tau), \\
a_{4}= & \sum_{y=1}^{4} r_{5-y} \omega^{y \alpha+1} \sin \left(\omega \tau-\frac{(y \alpha+1) \pi}{2}\right)+\omega X_{3} \cos (\omega \tau) .
\end{aligned}
$$

Then

$$
\operatorname{Re}\left[\left(\frac{d \lambda}{d \tau}\right)^{-1} \mid \tau=\tau_{j}, \omega=\omega_{0}\right]=\frac{a_{1} a_{3}+a_{2} a_{4}}{a_{3}^{2}+a_{4}^{2}} \neq 0
$$


The transversality condition holds, and Hopf bifurcation occurs at $\tau=\tau_{j}$. Hence proved.

R e m ark 2. A unique endemic equilibrium $E_{1}$ exist when $R_{0}>1$. If the time delay $\tau \in$ $\left[0, \tau_{0}\right)$ the endemic equilibrium $E_{1}$ is locally asymptotically stable. When the time delay $\tau$ passes through the critical value $\tau_{0}$, the endemic equilibrium $E_{1}$ will become unstable and a Hopf bifurcation occurs under some conditions. This biologically means as the time delay $\tau$ increases, the numbers of the uninfected population.

The next section deals with the sensitivity analysis of the basic reproduction number $R_{0}$.

\section{SENSITIVITY INDICES OF $\beta_{1}, \beta_{2}, \beta_{3}, \rho_{1}, \rho_{2}, \rho_{3}, \varphi$ and $\mu$ WITH $R_{0}$}

In this section, sensitivity analysis is performed to identify the important parameter which contributes to the variability in the outcome of the basic reproduction number based on their estimation uncertainty [42]. Partial rank correlation coefficient (PRCCs) is estimated between the values of $R_{0}$ and the values of each of the eight parameters, which is derived from the uncertainty analysis. The PRCCs value for $R_{0}$ and each of eight parameters enlisted in Table 3 and graphically represented in Fig. 3 .

The parameter with positive PRCCs is directly proportional to $R_{0}$, i.e. $\beta_{1}, \beta_{2}, \beta_{3}$ and $\varphi$, whereas the parameter with negative PRCCs is inversely proportional to $R_{0}$, i.e. $\rho_{1}, \rho_{2}, \rho_{3}$ and $\mu$. We observe that the parameter $\beta_{1}$ is highly correlated, and $\mu$ is the second highly correlated parameter. Hence we can conclude that $\beta_{1}$ and $\mu$ are the most important parameters in determining the $R_{0}$.

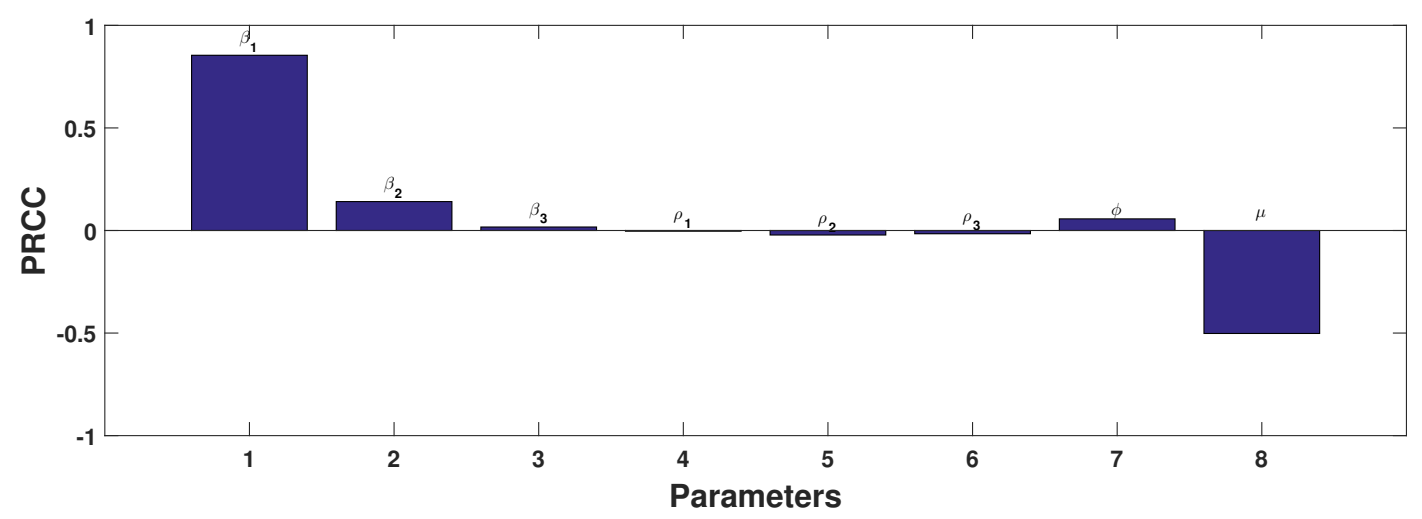

Fig. 2. The PRCCs between input parameters and output $R_{0}$.

\section{NUMERICAL SIMULATION AND DISCUSSIONS}

The calculations has been carried out using MATLAB (R2015a), in order to represent the major theoretical results of the delayed fractional order phase structured HIV model (2). Numerical simulations has been performed using predictor-corrector algorithm for solving fractional differential equations involving delay [43]. Here, the figures are plotted with the initial conditions as $S(0)=5000, I(0)=4000, K(0)=3000, H(0)=2000$ and $A(0)=1000$, with the delay term $\tau=4$.

- Fig. 3 has been plotted using the parameters listed in the Table 2 for different values of $\alpha$, where $R_{0}=0.0391<1$. In this scenario, it can be noticed that susceptible and acute phase solution curves tend to become constant, while other solution curves of three phase tend to zero. This biologically means that infective individual gradually decreases when $R_{0}<1$ and uninfected population survives for a better health life. 
Table 3. PRCCs for $R_{0}$ and eight input parameters

\begin{tabular}{|c|c|c|}
\hline S. No. & Parameter & PRCCs \\
\hline 1. & $\beta_{1}$ & 0.8543 \\
2. & $\beta_{2}$ & 0.1410 \\
3. & $\beta_{3}$ & 0.0170 \\
4. & $\rho_{1}$ & -0.0033 \\
5. & $\rho_{2}$ & -0.0220 \\
6. & $\rho_{3}$ & -0.0159 \\
7. & $\varphi$ & 0.0568 \\
8. & $\mu$ & -0.5020 \\
\hline
\end{tabular}

- Fig. 4 has been plotted using the parameters listed in the Table 2 except for $\beta_{1}=$ $0.00002, \beta_{2}=0.00005, \beta_{3}=0.00004, \rho_{1}=0.4, \sigma_{2}=0.09$, for different values of $\alpha$, where $R_{0}=1.9204>1$. In this case it can be observed that the solution curve of the all compartments attains a constant. This biologically means that infective individual survives when $R_{0}>1$.

It can be observed from Fig. $\underline{3}$ and Fig. 4 , that the solution trajectories of the fractional order system behave like its integer counterpart. The results show that order of the fractional derivative has a significant effect on the dynamic process. Also, from the results, it is obvious that the memory effect is zero for $\alpha=1$. When the fractional order $\alpha$ is decreased from 1 , the memory trace nonlinearly increases from 0 , and its dynamics strongly depends on time. The memory effect points out the difference between the derivatives of the fractional order and integer order [44]. In the following subsection we have analyzed the impact of three distinct treatments for our proposed model (2).

\subsection{Impact of treatment and its combinations}

Here, we discuss about the impact of treatment and its combination in consecutive phases. Fig. 5, Fig. 6 and Fig. 7 have been plotted with the same initial conditions and parameters used to plot Fig. $\overline{4}$, except for $\alpha=0.95$. In this study we have analyzed eight cases of treatment and its impact in the dynamics of the system (2), which are tabulated in Table 4 and the data has been figured out using the observation from Fig. 5 , Fig. 6 and Fig. 7.

In Table 4 the survival rate of population in the compartments under certain condition of treatment is denoted by $\checkmark$, while the non survival rate of population is denoted by $\times$. It can be observed that cases No. 1, 3 and 6 behave alike and cases No. 4, 5 and 7 also behave in a similar fashion. The most hazardous instance is cases No. 2 and 8 , where in the case No. 2 AIDS population survive, and in the case No. 8 symptomatic population survive. This is due to the zero treatment and treatment at last phase. It can be noted down that AIDS population attains a constant only when there is zero treatment and in other case of partial and full-fledged treatment the AIDS population tends to zero. From this we can conclude that the population will attain AIDS phase only when it is left without treatment. So the above analysis shows that 
PITCHAIMANI AND SARANYA DEVI

implementing treatment at the earlier phase works better than treatment at second and third phases.

Table 4. Treatment and its cases

\begin{tabular}{|c|c|c|c|c|c|c|}
\hline Case No. & Condition & $S(t)$ & $I(t)$ & $K(t)$ & $H(t)$ & $A(t)$ \\
\hline 1. & $\rho_{1}=\rho_{2}=\rho_{3}=0.99$ & $\checkmark$ & $\checkmark$ & $\times$ & $\times$ & $\times$ \\
\hline 2. & $\rho_{1}=\rho_{2}=\rho_{3}=0$ & $\checkmark$ & $\checkmark$ & $\checkmark$ & $\checkmark$ & $\checkmark$ \\
\hline 3. & $\rho_{1}=\rho_{2}=0.99, \rho_{3}=0$ & $\checkmark$ & $\checkmark$ & $\times$ & $\times$ & $\times$ \\
\hline 4. & $\rho_{1}=0, \rho_{2}=\rho_{3}=0.99$ & $\checkmark$ & $\checkmark$ & $\checkmark$ & $\times$ & $\times$ \\
\hline 5. & $\rho_{1}=0.99, \rho_{2}=0, \rho_{3}=0.99$ & $\checkmark$ & $\checkmark$ & $\checkmark$ & $\times$ & $\times$ \\
\hline 6. & $\rho_{1}=0.99, \rho_{2}=\rho_{3}=0$ & $\checkmark$ & $\checkmark$ & $\times$ & $\times$ & $\times$ \\
\hline 7. & $\rho_{1}=0, \rho_{2}=0.99, \rho_{3}=0$ & $\checkmark$ & $\checkmark$ & $\checkmark$ & $\times$ & $\times$ \\
\hline 8. & $\rho_{1}=0, \rho_{2}=0, \rho_{3}=0.99$ & $\checkmark$ & $\checkmark$ & $\checkmark$ & $\checkmark$ & $\times$ \\
\hline
\end{tabular}

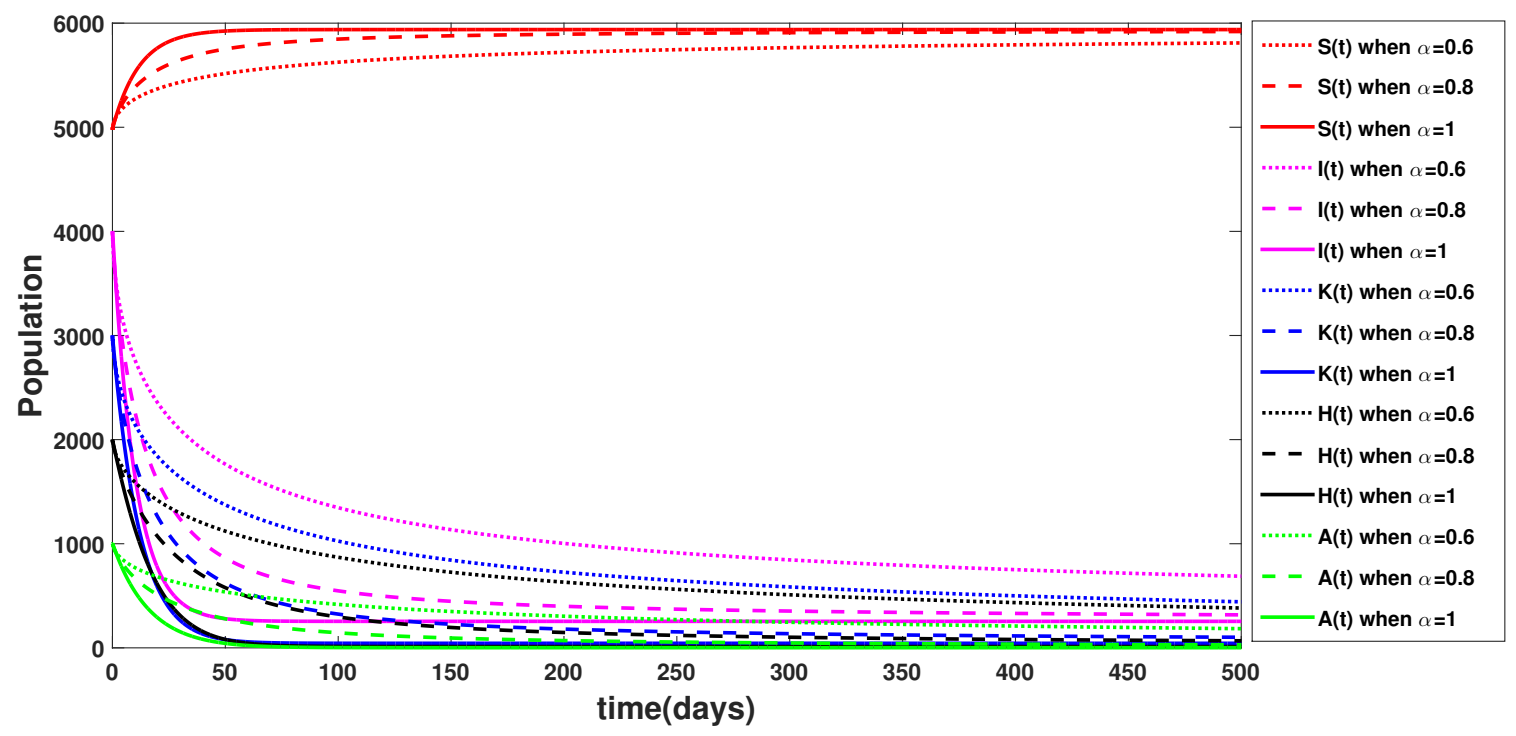

Fig. 3. The graph denotes trajectories of $S(t), I(t), K(t), H(t)$ and $A(t)$ versus time $t$ of system (2), where $R_{0}=0.03906<1$. 


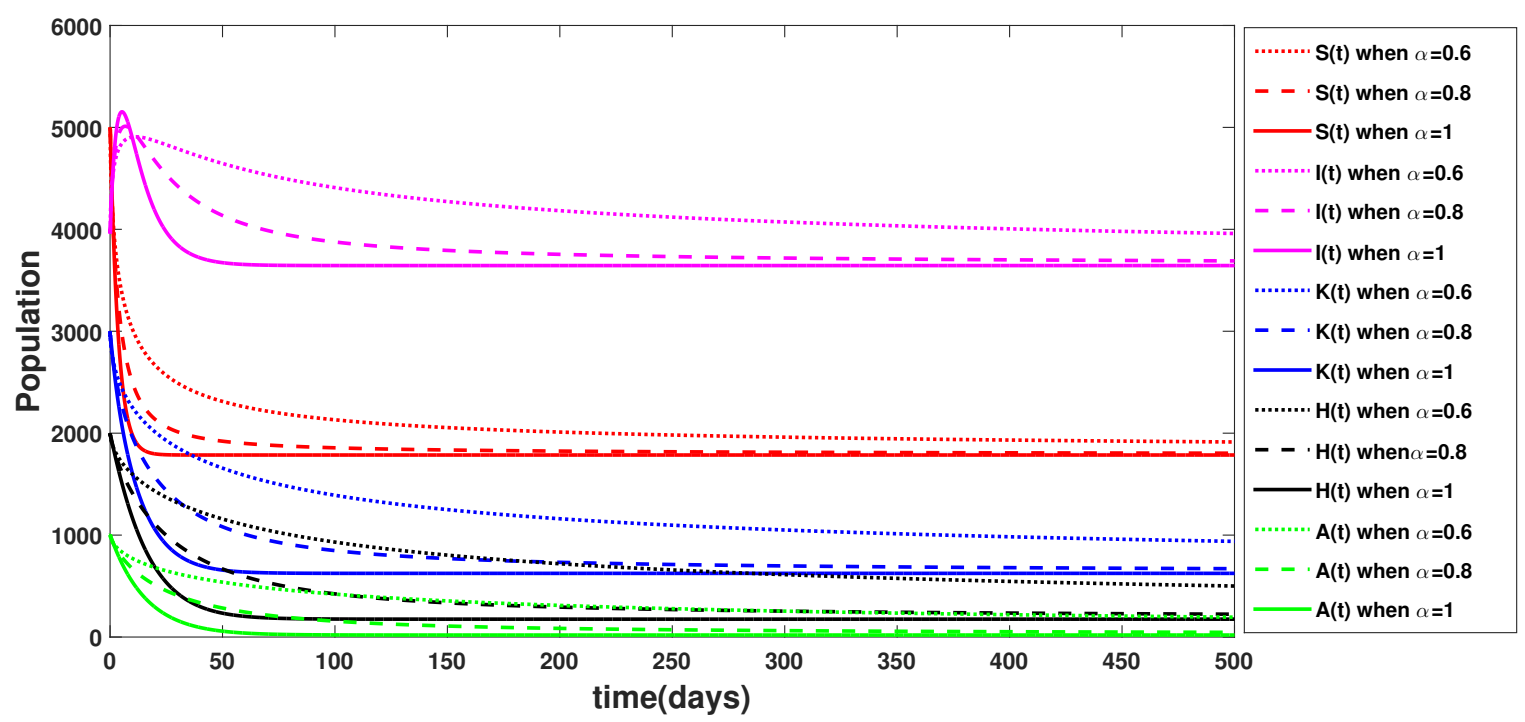

Fig. 4. The graph denotes trajectories of $S(t), I(t), K(t), H(t)$ and $A(t)$ versus time $t$ of system (2), where $R_{0}=1.9234>1$.

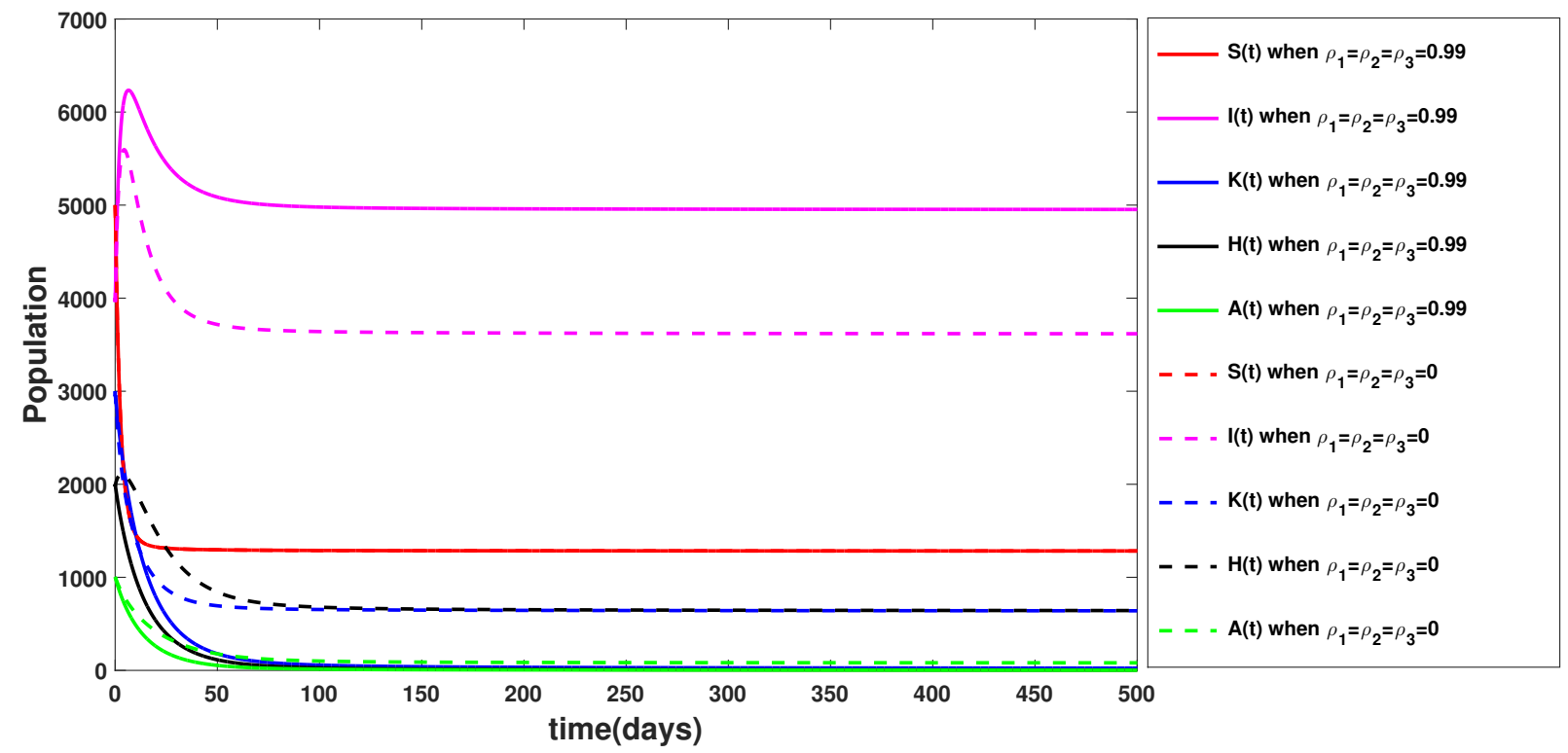

Fig. 5. The figure denotes the impact of treatment on infectives for case No.1 $\left(\rho_{1}=\rho_{2}=\rho_{3}=0.99\right)$ and case No.2 $\left(\rho_{1}=\rho_{2}=\rho_{3}=0\right)$. 


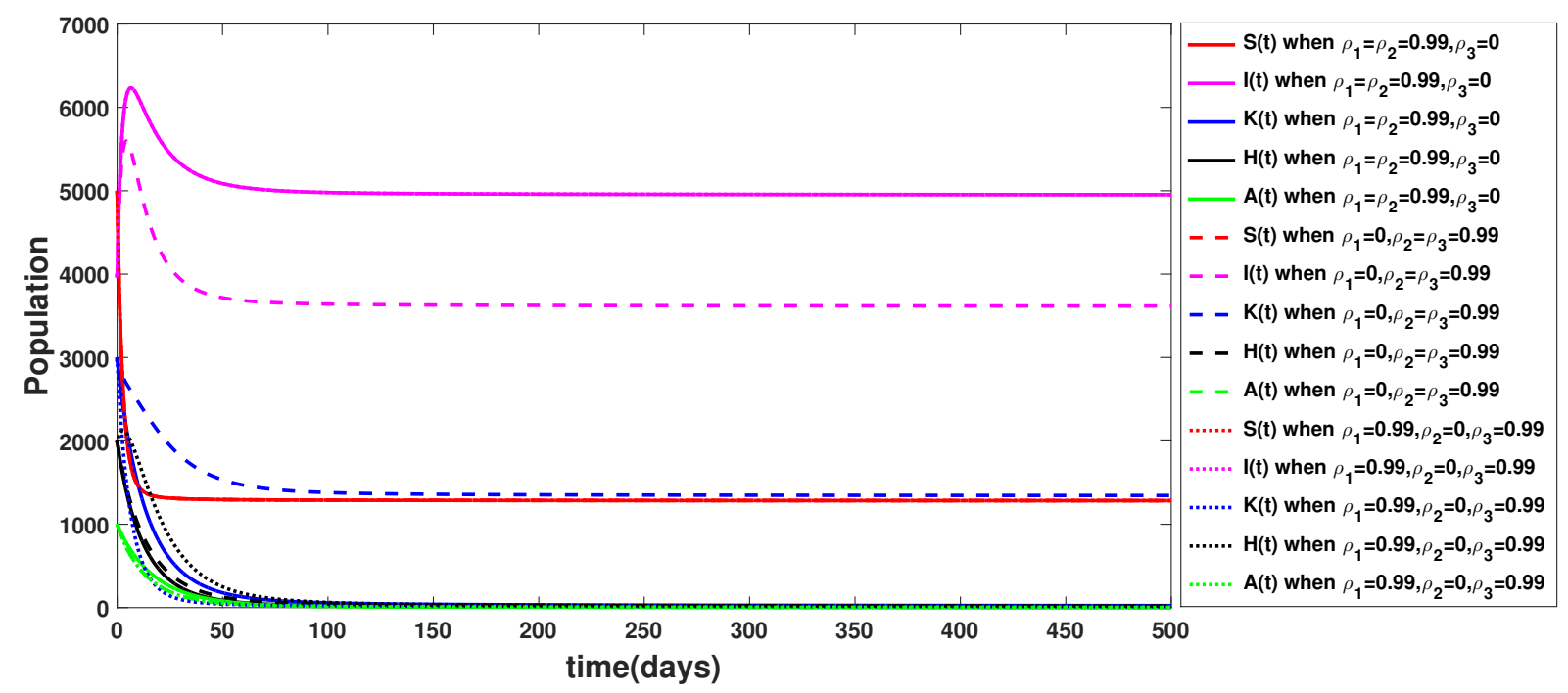

Fig. 6. The figure denotes the impact of treatment on infectives for case No.3 $\left(\rho_{1}=\rho_{2}=0.99, \rho_{3}=0\right)$, case No.4 $\left(\rho_{1}=0, \rho_{2}=\rho_{3}=0.99\right)$ and case No.5 $\left(\rho_{1}=0.99, \rho_{2}=0, \rho_{3}=0.99\right)$.

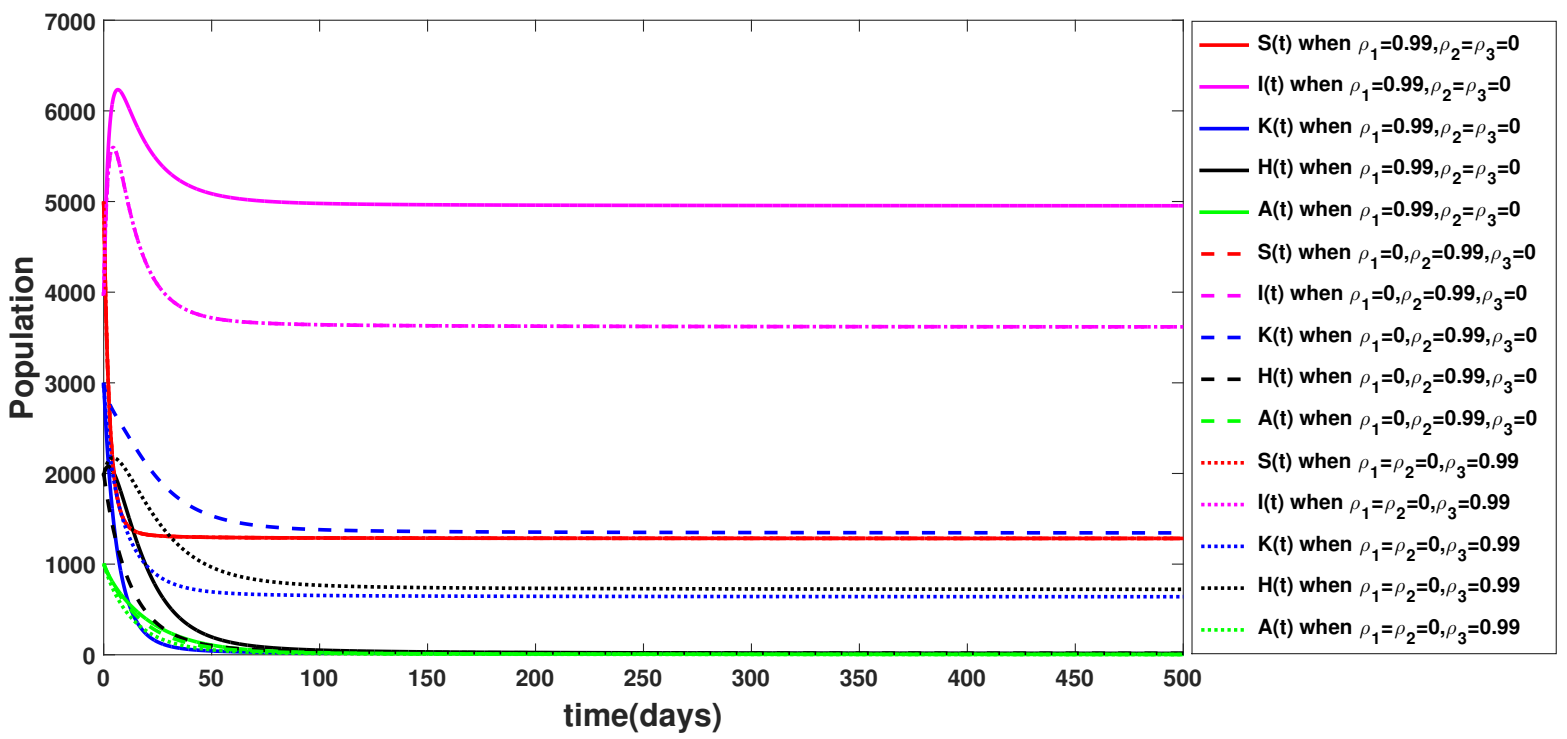

Fig. 7. The above figure denotes the impact of treatment on infectives for case No.6 $\left(\rho_{1}=0.99, \rho_{2}=\rho_{3}=0\right)$, case No.7 $\left(\rho_{1}=0, \rho_{2}=0.99, \rho_{3}=0\right)$ and case No.8 $\left(\rho_{1}=\rho_{2}=0, \rho_{3}=0.99\right)$. 


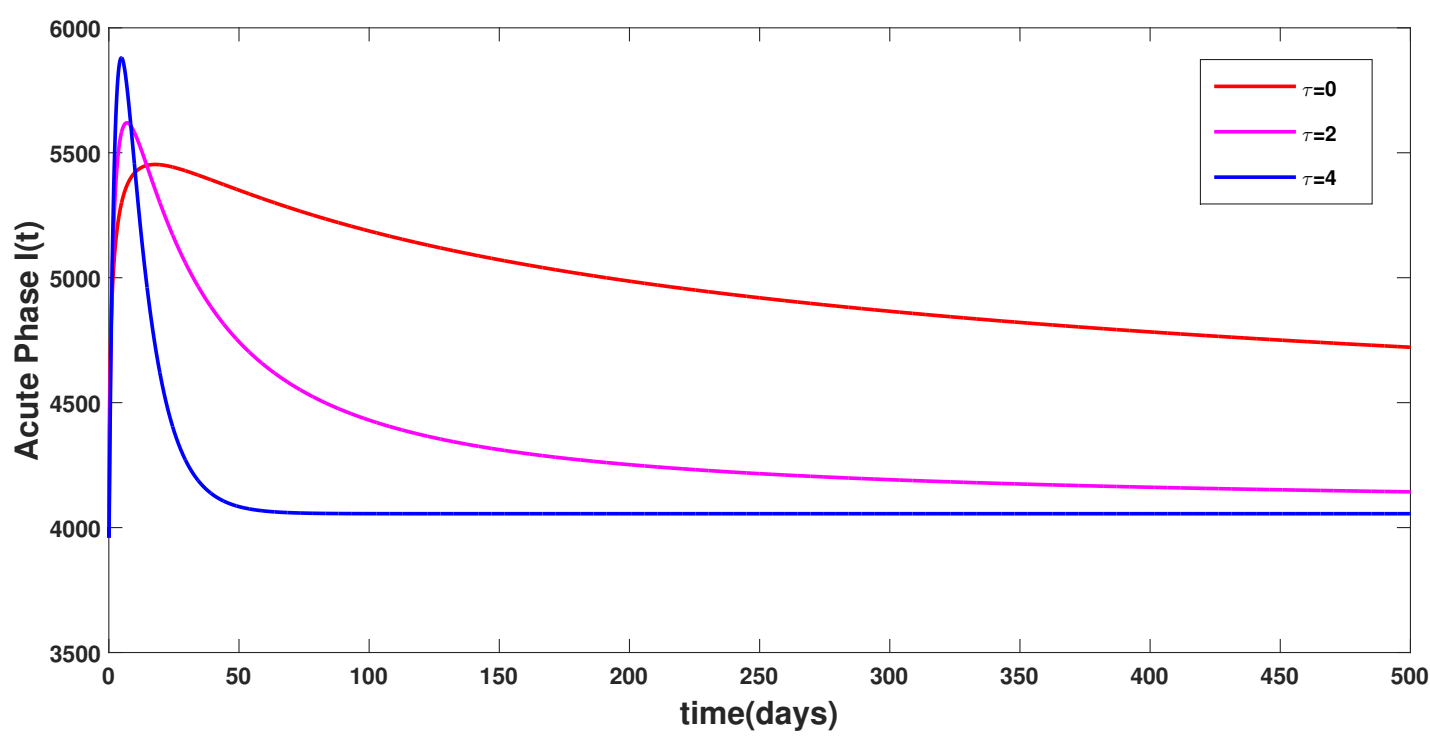

Fig. 8. The above figure denotes the impact of the delay parameter $\tau$ at the acute phase when $R_{0}>1$. It can be observed that the infected population at the acute phase decreases as the delay parameter $\tau$ increases, which biologically denotes that the delay parameter $\tau$ plays a vital role in disease control.

\section{PARAMETER ESTIMATION}

The baseline parameter values and initial population values obtained through curve fitting are presented at Table 5 and Table 6 . The full list of parameter ranges used in the simulation is given in Table 2 .

\subsection{Data}

The data were obtained from World Health Organization [45]. The data used represent new HIV infection in Afghanistan, Australia, France, Italy, Netherlands and New Zealand. Data are collected routinely on a yearly basis and was retrieved for the period beginning January 2000 to December 2019. The pictorial representation of the raw data is given in Figures $9-14$. We fit the model (2) to yearly new HIV infected population for these six countries. The notified cases have high risk of spreading infection and thus it is convenient to fit the HIV infected cases to the reported data.

\subsection{Curve fitting}

In this section, we fit system (2) to data to determine the trend of HIV in populations. Curve fitting is a process that allows us to quantitatively estimate the trend of the outcomes. The curve fitting process fits equations of approximating curves to the raw field data. However, for a given set of data, the fitting curves of a given type are generally not unique. Thus, a curve with a minimal deviation from all data points is desired. This best fitting curve can be obtained by the method of least squares. In this method, the parameters not known are approximated through minimization of the sum of the squared deviations between the data and the model. It minimizes the sum of squared distances between the observed values and the model values. This can be mathematically expressed as

$$
R S S=\sum_{i=1}^{n} \Theta_{i}^{2}=\sum_{i=1}^{n}\left(Z_{i}-\hat{Z}\right)^{2}
$$


where $\Theta_{i}=\left(Z_{i}-\hat{Z}\right)$ and $\mathrm{n}$ refers to the data points and RSS refers to the sum of square error estimate which is assumed to follow a normal distribution. The following parameters were fixed at the following values: $\beta_{1}=0.00002, \beta_{2}=0.00005, \beta_{3}=0.00004, \mu=3.96, \sigma_{1}=$ $0.03, \sigma_{2}=0.09, \sigma_{3}=0.01, \rho_{1}=0.65, \rho_{2}=0.5, \rho_{3}=0.1, d=0.001, S(0)=5000, I(0)=$ $4000, K(0)=3000, H(0)=2000$ and $A(0)=1000$. The parameter ranges/values in parameter values in Table 2 are used in the curve fitting and the resulting estimated parameter values and initial values are presented in Table 5 and Table 6. Figures 9-14 denote the data fitted to system (2) for the reported new cases of HIV infection in Afghanistan, Australia, France, Italy, Netherlands and New Zealand. We observe that the model fits well with the data.

Table 5. Estimated parameter values of the model (2) for the countries Afghanistan, Australia, France, Italy, Netherlands and New Zealand

\begin{tabular}{|c|c|c|c|c|c|c|}
\hline Par. & Afghanistan & Australia & France & Italy & Netherlands & New Zealand \\
\hline$\beta_{1}$ & $1.963 \times 10^{-9}$ & $2.142 \times 10^{-9}$ & $2.023 \times 10^{-10}$ & $2.020 \times 10^{-10}$ & $2.156 \times 10^{-9}$ & $2.035 \times 10^{-8}$ \\
\hline$\beta_{2}$ & $4.997 \times 10^{-9}$ & $5.018 \times 10^{-9}$ & $5.046 \times 10^{-10}$ & $5.193 \times 10^{-10}$ & $5 \times 10^{-9}$ & $5.133 \times 10^{-8}$ \\
\hline$\beta_{3}$ & $3.710 \times 10^{-9}$ & $4.082 \times 10^{-9}$ & $3.932 \times 10^{-10}$ & $4.061 \times 10^{-10}$ & $4.136 \times 10^{-9}$ & $4.033 \times 10^{-8}$ \\
\hline$\mu$. & $1.080 \times 10^{-5}$ & $5.575 \times 10^{-6}$ & $5.128 \times 10^{-7}$ & $4.179 \times 10^{-7}$ & $5.215 \times 10^{-6}$ & $7.366 \times 10^{-5}$ \\
\hline$d$ & $1.011 \times 10^{-7}$ & $9.68 \times 10^{-8}$ & $9.981 \times 10^{-9}$ & $9.801 \times 10^{-9}$ & $9.713 \times 10^{-7}$ & $9.878 \times 10^{-7}$ \\
\hline$\sigma_{1}$ & $7.796 \times 10^{-6}$ & $7.992 \times 10^{-6}$ & $8.016 \times 10^{-7}$ & $8.310 \times 10^{-7}$ & $8.046 \times 10^{-6}$ & $8.272 \times 10^{-5}$ \\
\hline$\sigma_{2}$ & $8.900 \times 10^{-6}$ & $9.546 \times 10^{-6}$ & $8.825 \times 10^{-7}$ & $8.752 \times 10^{-7}$ & $9.548 \times 10^{-6}$ & $8.769 \times 10^{-5}$ \\
\hline$\sigma_{3}$ & $9.363 \times 10^{-7}$ & $1.004 \times 10^{-6}$ & $9.890 \times 10^{-8}$ & $1.013 \times 10^{-7}$ & $1.006 \times 10^{-6}$ & $1.008 \times 10^{-5}$ \\
\hline$\rho_{1}$ & $3.896 \times 10^{-5}$ & $3.879 \times 10^{-5}$ & $4.532 \times 10^{-6}$ & $4.667 \times 10^{-6}$ & $3.844 \times 10^{-5}$ & $4.035 \times 10^{-4}$ \\
\hline$\rho_{2}$ & $4.952 \times 10^{-5}$ & $4.919 \times 10^{-5}$ & $5.080 \times 10^{-6}$ & $4.927 \times 10^{-6}$ & $4.909 \times 10^{-5}$ & $5.032 \times 10^{-4}$ \\
\hline$\rho_{3}$ & $9.889 \times 10^{-6}$ & $1.046 \times 10^{-5}$ & $1.020 \times 10^{-6}$ & $1.015 \times 10^{-6}$ & $1.048 \times 10^{-5}$ & $9.892 \times 10^{-5}$ \\
\hline
\end{tabular}




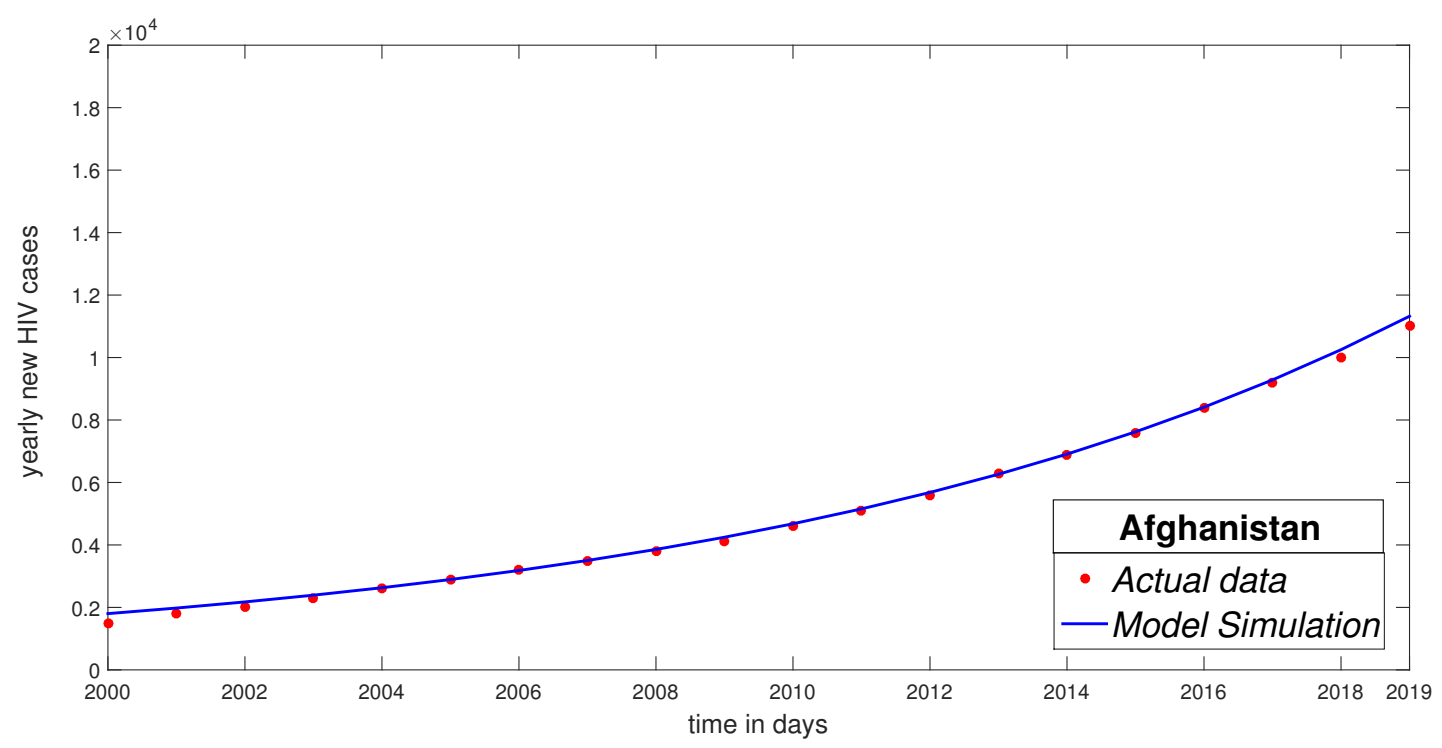

Fig. 9. Denote the data fitted to system (2) for the reported new cases of HIV infection in Afghanistan.

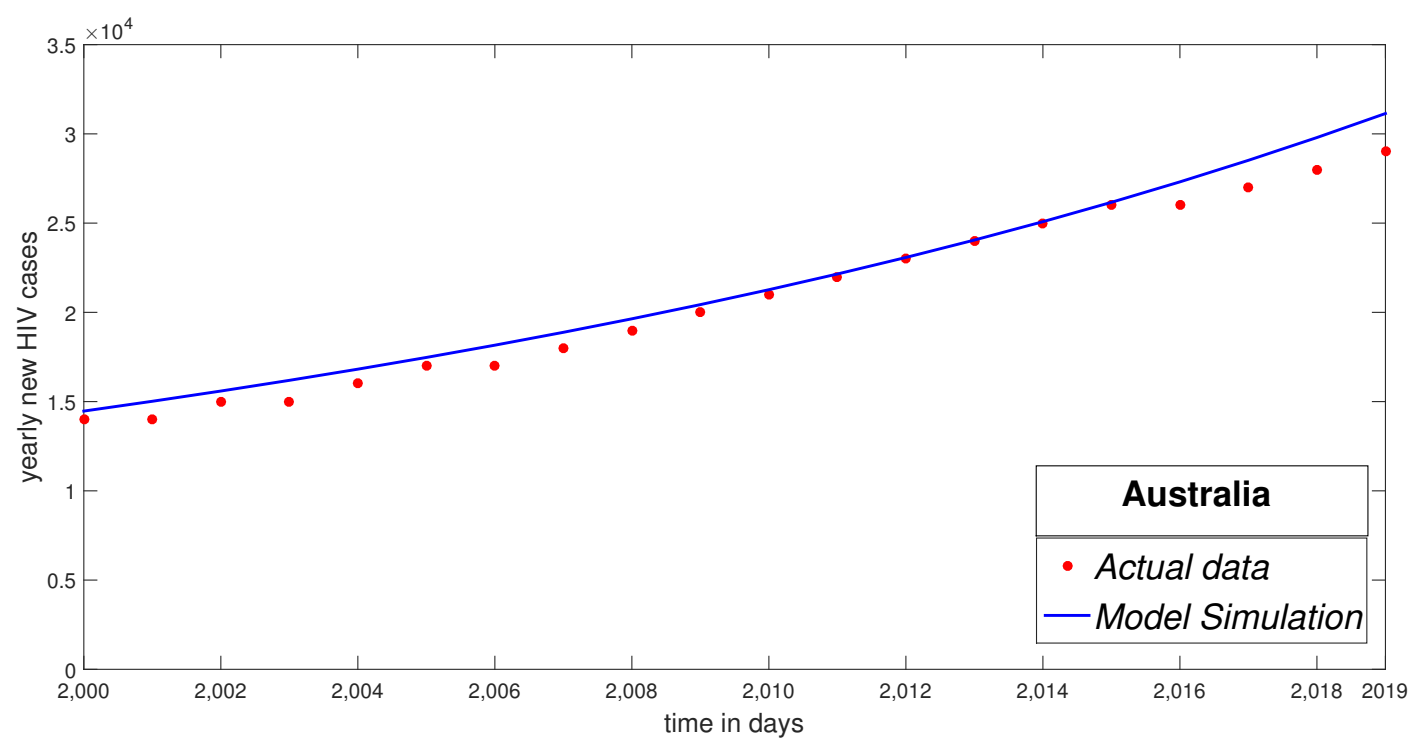

Fig. 10. Denote the data fitted to system (2) for the reported new cases of HIV infection in Australia. 


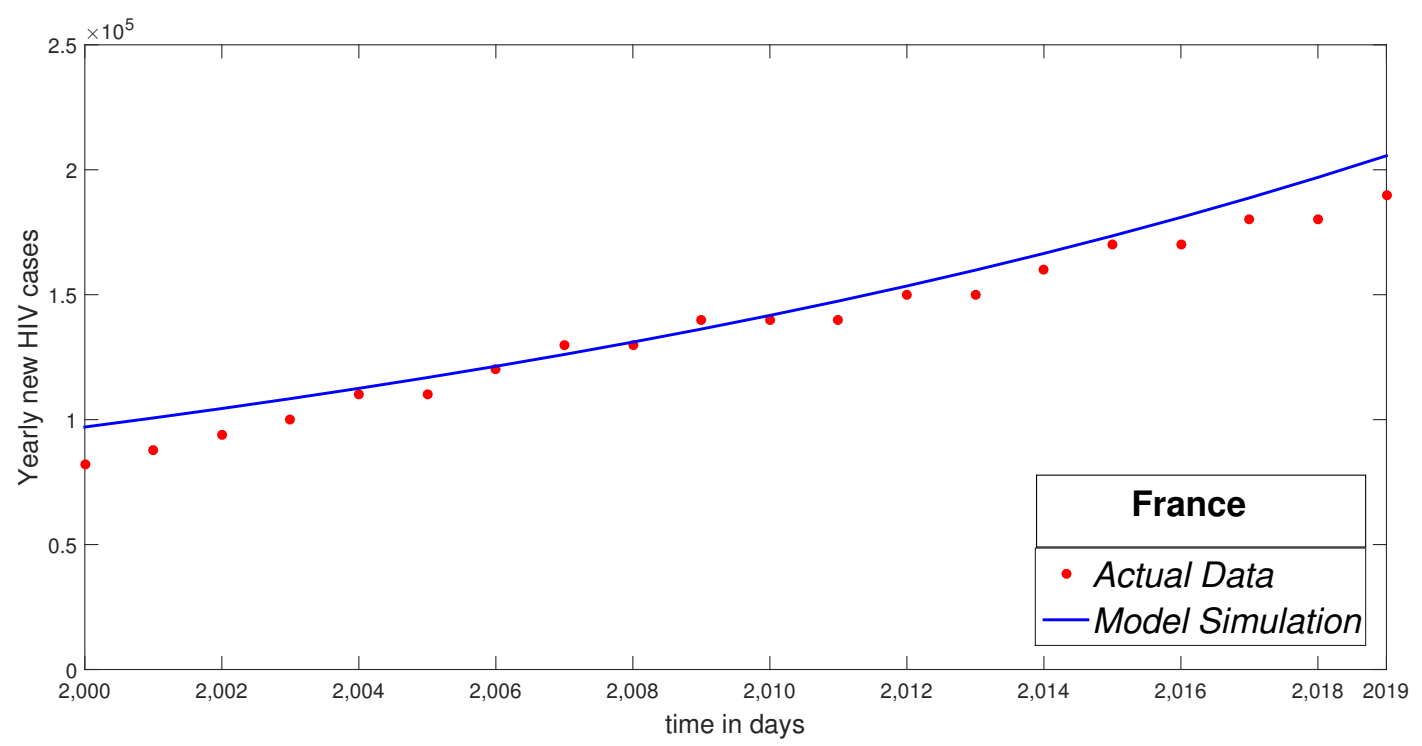

Fig. 11. Denote the data fitted to system (2) for the reported new cases of HIV infection in France.

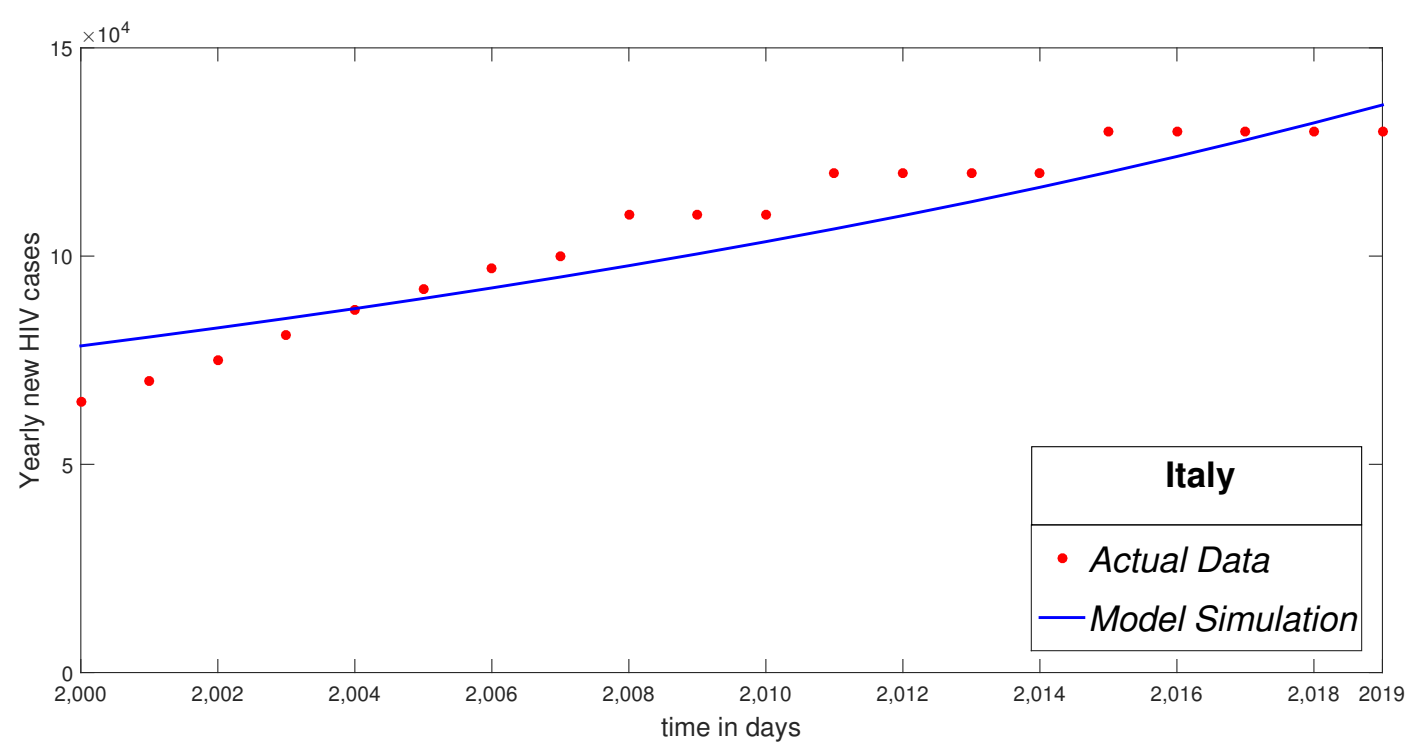

Fig. 12. Denote the data fitted to system (2) for the reported new cases of HIV infection in Italy. 


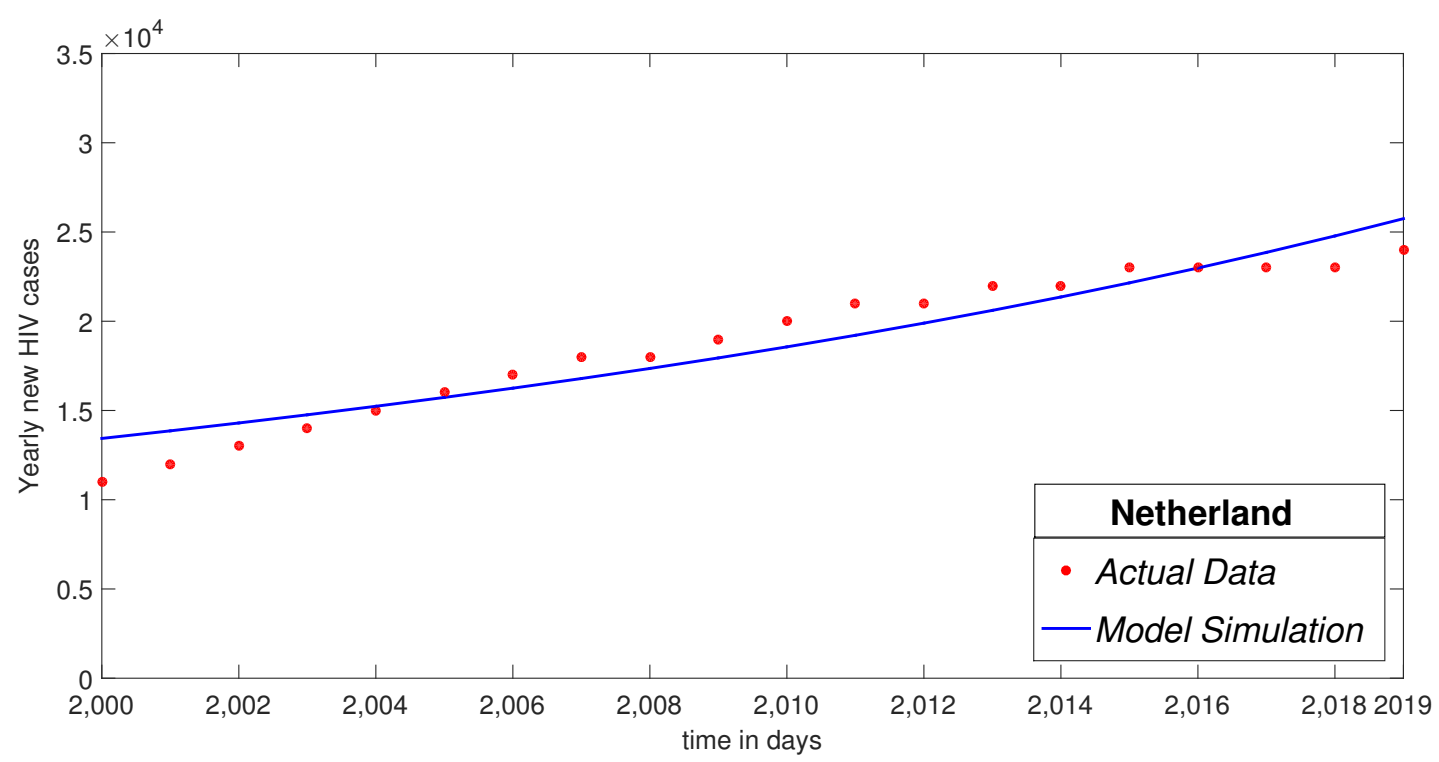

Fig. 13. Denote the data fitted to system (2) for the reported new cases of HIV infection in Netherlands.

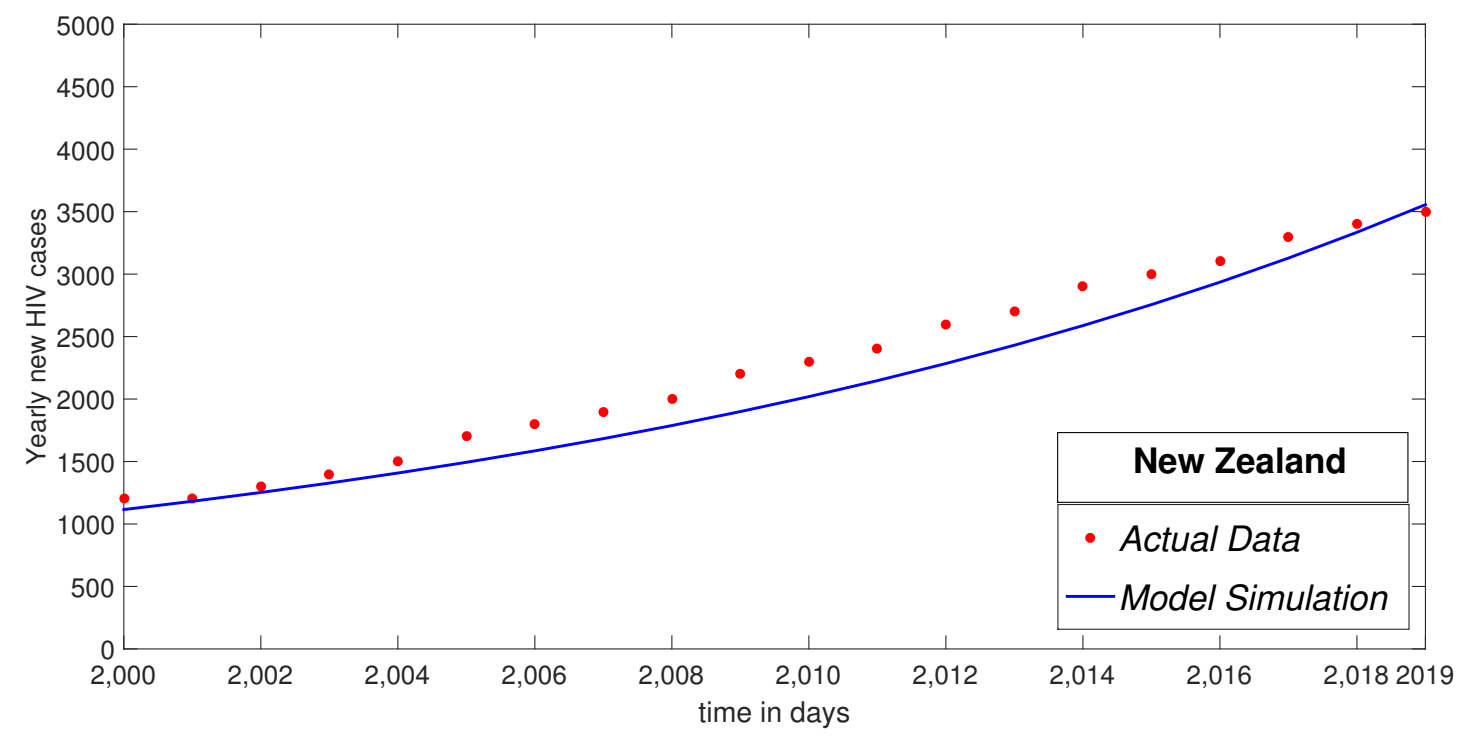

Fig. 14. Denote the data fitted to system (2) for the reported new cases of HIV infection in New Zealand. The baseline parameter values and initial population values obtained from the curve fitting are given in Table 5 and Table 6. 
Table 6. Estimated initial population values of the model (2) for the countries Afghanistan, Australia, France, Italy, Netherlands and New Zealand

\begin{tabular}{|c|c|c|c|c|c|c|}
\hline Initial Population & Afghanistan & Australia & France & Italy & Netherlands & New Zealand \\
\hline$S(0)$ & 1551 & 14055 & 82056 & 65004 & 11056 & 1221 \\
\hline$I(0)$ & 1235 & 302 & 50453 & 46034 & 9876 & 987 \\
\hline$K(0)$ & 1455 & 1028 & 30765 & 33564 & 1543 & 675 \\
\hline$H(0)$ & 176 & 756 & 1098 & 3445 & 3456 & 445 \\
\hline$A(0)$ & 10 & 59 & 345 & 245 & 235 & 24 \\
\hline
\end{tabular}

\section{CONCLUSIONS}

A delayed fractional order phase structured HIV model with three phases, and three distinct therapies have been studied to analyze the dynamics of the disease. In this article, the course of infection have been categorized into the acute, asymptomatic, and symptomatic phases. By all sorts of treatment methods, individuals with the asymptomatic, symptomatic phases can be transformed into acute individuals. The dynamical behavior of the system (2) can be determined by its basic reproduction number $R_{0}$, i.e., If $R_{0}<1$, the disease-free equilibrium is globally stable. If $R_{0}>1$, the disease persists, and the unique endemic equilibrium is globally asymptotically stable. Thus for control of disease in a population, it is generally accepted that it is desirable for $R_{0}$ to be as small as possible. Particularly, it is desired, if possible, for $R_{0}$ to be less than one. The treatment rate $\rho_{1}, \rho_{2}$ and $\rho_{3}$ inversely proportional to $R_{0}$, it is clear that in absence of treatment $R_{0}>1$. This ensures that in some cases, it is possible that the treatment can be used to make $E_{0}$ stable when it would be unstable in the absence of treatment. On the other hand, if $R_{0}<1$, then by making $R_{0}$ sufficiently large, $E_{0}$ can be switched from stable to unstable, causing the disease to persist in the population when it otherwise would have died out. This situation reveals the impact of treatment on disease dynamics.

The novelty of the work lies in the incorporation of two types of memories, i.e. time delay and fractional order in the proposed model (2), which makes it more realistic and enrich the dynamics of the proposed model (2) when compared to the HIV model analyzed by swarnali sharma and samanta [4]. In addition, we have also analyzed eight treatment cases which has been never discussed before in the existing literature. It can be observed from Fig. (3) and Fig. (4) that the solution trajectories of the fractional order delay system (2) becomes stable only if its corresponding integer order solution trajectory gets stable. The main contributions of the work are highlighted as follows,

1. We have computed basic reproduction number $R_{0}$, and the local stability of the equilibria for the system (2) has been discussed from the existing theories.

2. Predictor-corrector algorithm has been adapted to solve the fractional order delay differential equation and to plot the solution trajectories of the non linear fractional delay system (2).

3. Sensitivity analysis for the basic reproduction number $R_{0}$ has been performed. 
4. Parameters have been estimated to fit yearly data for countries Afghanistan, Australia, France, Italy, Netherlands and New Zealand for system (2).

The above contributions help us to view the dynamics of the proposed model. The ultimate aim is to capture the most sensitive phase to understand the dynamics. It is a well known fact that HIV is an incurable epidemic, the only way to increase the life span is to undergo suitable treatment at the earliest phase. This helps to survive in a long run. In numerical analysis, (see. Figures 5-7) we have depicted the graph for eight cases of treatment, from which we conclude that providing full-fledged treatment at the earlier stage (acute phase) works better than other stages. In case if the person is aware of HIV infection at phase 2 or phase 3 (asymptomatic phase or symptomatic phase), it is better to treat the infected individual at that stage to maintain his health at the same phase of disease to avoid the infection progressing to AIDS.

\section{FUTURE CHALLENGES}

The limitations in the present study have been addressed in this section. Such limitations will serve as the foundations for potential subsequent work in this area. First of all, we list some of the major constraints of the study as follows:

i. The proposed model can also be generalized for $\mathrm{n}$ compartments with $\mathrm{n}$ distinct delays and $\mathrm{n}$ phases.

ii. It can also be developed by the incorporation of stochasticity into the model which will result as the HIV model with fractional stochastic delay.

iii. The proposed model can also be analyzed with the different incidence rates and $n$ parameter bifurcation analysis.

iv. Frequency-dependent phase structured HIV model can be developed to compare its behavior with our proposed density-dependent HIV model.

Therefore, the study suggests many paths for future research from the above discussion. The present study therefore serves as a new gateway for immunologists, eco-epidemiologists, and mathematicians working in disease modeling. The present study helps us to view the impact of memory and treatment in HIV modeling.

The University of Madras has supported this work through University Research Fellowship to Ms. A. Saranya Devi. 


\section{THE VALUES OF POLYNOMIAL COEFFICIENTS USED IN BIFURCATION ANALYSIS}

The values of polynomial coefficients used in bifurcation analysis (see section 2.5 ) are as follows:

$$
\begin{aligned}
& q_{1}=H_{1} \beta_{3}+I_{1} \beta_{1}+K_{1} \beta_{2}+d+5 \mu+\delta_{1}+\delta_{2}+\delta_{3}, \\
& r_{1}=-\beta_{1} S_{1}, \\
& q_{2}=\delta_{1}\left[3 \mu+H_{1} \beta_{3}+I_{1} \beta_{1}+K_{1} \beta_{2}\right]+\delta_{2}\left[3 \mu+\delta_{1}+H_{1} \beta_{3}+K_{1} \beta_{2}\right] \\
& +\delta_{3}\left[3 \mu+\delta_{2}+\delta_{3}+H_{1} \beta_{3}+2 I_{1} \beta_{1}\right]+3 \mu\left[2 \mu+H_{1} \beta_{3}+I_{1} \beta_{1}+K_{1} \beta_{2}\right] \\
& +(d+\mu)\left[4 \mu+\delta_{1}+\delta_{2}+\delta_{3}+H_{1} \beta_{3}+K_{1} \beta_{2}\right] \text {, } \\
& r_{2}=-S_{1}\left[\beta_{1}\left(3 \mu+\delta_{2}+\delta_{3}+d+\mu\right)+\beta_{2} \delta_{1}\right] \text {, } \\
& q_{3}=\left[\left(H_{1} \beta_{3}+I_{1} \beta_{1}+K_{1} \beta_{2}+d+3 \mu+\delta_{3}\right) \delta_{2}+\left(H_{1} \beta_{3}+I_{1} \beta_{1}+K_{1} \beta_{2}+d+3 \mu\right) \delta_{3}\right. \\
& \left.+3 \mu^{2}+2 \mu H_{1} \beta_{3}+2 \mu K_{1} \beta_{2}+\left(H_{1} \beta_{3}+I_{1} \beta_{1}+K_{1} \beta_{2}+3 \mu\right)(d+\mu)+2 \mu I_{1} \beta_{1}\right] \delta_{1} \\
& +\left[\left(H_{1} \beta_{3}+I_{1} \beta_{1}+K_{1} \beta_{2}+d+3 \mu\right) \delta_{3}+3 \mu^{2}+2 \mu H_{1} \beta_{3}+2 \mu K_{1} \beta_{2}\right. \\
& \left.+\left(H_{1} \beta_{3}+I_{1} \beta_{1}+K_{1} \beta_{2}+3 \mu\right)(d+\mu)+2 \mu I_{1} \beta_{1}\right] \delta_{2} \\
& +\left[3 \mu^{2}+2 \mu H_{1} \beta_{3}+2 \mu K_{1} \beta_{2}+\left(H_{1} \beta_{3}+I_{1} \beta_{1}+K_{1} \beta_{2}+3 \mu\right)(d+\mu)+2 \mu I_{1} \beta_{1}\right] \delta_{3} \\
& +3 \mu^{2}\left(I_{1} \beta_{1}+H_{1} \beta_{3}+K_{1} \beta_{2}\right)+4 \mu^{3}+\left[3 \mu\left(I_{1} \beta_{1}+H_{1} \beta_{3}+K_{1} \beta_{2}\right)+6 \mu^{2}\right](d+\mu), \\
& r_{3}=\left[-\delta_{3} \beta_{2} S_{1}-\beta_{2} S_{1}(d+\mu)-2 S_{1} \mu \beta_{2}-\delta_{2} S_{1} \beta_{3}\right] \delta_{1} \\
& -\left[\beta_{1} S_{1}(d+\mu)+\delta_{3} \beta_{1} S_{1}+2 \mu \beta_{1} S_{1}\right] \delta_{2}-\left[\beta_{1} S_{1}(d+\mu)+2 \mu \beta_{1} S_{1}\right] \delta_{3} \\
& -3 \mu \beta_{1} S_{1}(d+\mu)-3 \mu^{2} \beta_{1} S_{1} \\
& q_{4}=\left[\left(H_{1} \beta_{3}+I_{1} \beta_{1}+K_{1} \beta_{2}+d+2 \mu\right) \delta_{3}+\left(H_{1} \beta_{3}+I_{1} \beta_{1}+K_{1} \beta_{2}+2 \mu\right)(d+\mu)\right. \\
& \left.+\mu H_{1} \beta_{3}+\mu I_{1} \beta_{1}+\mu K_{1} \beta_{2}+\mu^{2}\right] \delta_{1} \delta_{2}+\left[\left(H_{1} \beta_{3}+I_{1} \beta_{1}+K_{1} \beta_{2}+2 \mu\right)(d+\mu)\right. \\
& \left.+\mu H_{1} \beta_{3}+\mu I_{1} \beta_{1}+\mu K_{1} \beta_{2}+\mu^{2}\right]\left(\delta_{3} \delta_{1}+\delta_{3} \delta_{2}\right)+\left[\mu^{2}\left(H_{1} \beta_{3}+I_{1} \beta_{1}+K_{1} \beta_{2}\right)+\mu^{3}\right. \\
& \left.+\left(2 \mu H_{1} \beta_{3}+2 \mu I_{1} \beta_{1}+2 \mu K_{1} \beta_{2}+3 \mu^{2}\right)(d+\mu)\right]\left(\delta_{1}+\delta_{2}+\delta_{3}\right) \\
& +\left[\mu^{3}\left(H_{1} \beta_{3}+I_{1} \beta_{1}+K_{1} \beta_{2}+\mu\right)+3 \mu^{2}\left(H_{1} \beta_{3}+I_{1} \beta_{1}+K_{1} \beta_{2}+\mu\right)(d+m u)\right], \\
& r_{4}=-\left[\mu\left(\beta_{3} \delta_{2}+\beta_{2} \delta_{3}+\mu \beta_{2}\right)+(d+\mu)\left(\beta_{3} \delta_{2}+\beta_{2} \delta_{3}+2 \mu \beta_{2}\right)\right] S_{1} \delta_{1} \\
& -\left[\left(\beta_{1} S_{1}(d+2 \mu)+2 \mu S_{1} \beta_{1}(d+\mu)-S_{1} \mu^{2} \beta_{1}\right] \delta_{2}\right. \\
& -\left[\mu^{2} S_{1} \beta_{1}-2 \mu S_{1} \beta_{1}(d+\mu)\right] \delta_{3}-\mu^{3} S_{1} \beta_{1}-3 \mu^{2} S_{1} \beta_{1}(d+\mu), \\
& q_{5}=\left(H_{1} \beta_{3}+I_{1} \beta_{1}+K_{1} \beta_{2}+m u\right)(d+\mu)\left[\delta_{1} \delta_{3}+\delta_{2} \delta_{3}+\mu \delta_{1} \delta_{2}+\mu \delta_{1} \delta_{3}\right. \\
& \left.+\mu^{2} \delta_{1}+\mu^{2} \delta_{2}+\mu^{2}+\mu^{3}\right] \\
& r_{5}=-S_{1}(d+\mu) \mu\left[\left(\beta_{3} \delta_{2}+\beta_{2} \delta_{3}+\mu \beta_{2}\right) \delta_{1}+2\left(\beta_{1} \delta_{3}+\mu \beta_{1}\right) \delta_{2}+2 \mu \beta_{1} \delta_{3}+2 m u^{2} \beta_{1}\right] \text {, } \\
& h_{1}=2 q_{1} \cos \left(\frac{\alpha \pi}{2}\right) \text {, } \\
& h_{2}=2 q_{2} \cos (\alpha \pi)-r_{1}^{2}\left(4 \sin ^{2}(\alpha \pi) \cos ^{2}(\alpha \pi)+4 D_{3}^{2}+1\right)+q_{1}^{2}, \\
& h_{3}=-2 q_{3} \cos \left(\frac{7 \alpha \pi}{2}\right)-8 D_{3}^{2} r_{1} r_{2} \cos \left(\frac{3 \alpha \pi}{2}\right) \cos (2 \alpha \pi) \\
& +\left(q_{1} q_{2}-r_{1} r_{2}\right)\left(4 \cos (\alpha \pi) \cos \left(\frac{\alpha \pi}{2}\right)-2 \cos \left(\frac{3 \alpha \pi}{2}\right)\right), \\
& h_{4}=-8 r_{1} r_{3} D_{3}^{2} \cos (\alpha \pi) \cos (2 \alpha \pi)+2 \cos (\alpha \pi)\left(q_{1} q_{3}-r_{1} r_{3}\right) \\
& +2 q_{4} \cos (2 \alpha \pi)+q_{2}^{2}-r_{2}^{2}-4 r_{2}^{2} D_{3}^{2} \cos ^{2}\left(\frac{3 \alpha \pi}{2}\right),
\end{aligned}
$$




$$
\begin{aligned}
h_{5} & =-8 r_{2} r_{3} D_{3}^{2} \cos \left(\frac{3 \alpha \pi}{2}\right) \cos (\alpha \pi)+4 \cos (\alpha \pi) \cos \left(\frac{\alpha \pi}{2}\right)\left(q_{1} q_{4}-r_{1} r_{4}\right) \\
& +2 \cos \left(\frac{\alpha \pi}{2}\right)\left(q_{2} q_{3}-r_{2} r_{3}-q_{1} q_{4}-r_{1} r_{4}\right)-8 r_{1} r_{4} D_{3}^{2} \cos \left(\frac{\alpha \pi}{2}\right) \cos (2 \alpha \pi), \\
h_{6} & =-r_{3}^{2}+q_{3}^{2}-2 r_{2} r_{4} \cos (\alpha \pi)+2 q_{1} q_{5} \cos (2 \alpha \pi)+2 q_{2} q_{4} \cos (\alpha \pi) \\
& -8 D_{3}^{2} r_{2} r_{4} \cos \left(\frac{3 \alpha \pi}{2}\right) \cos \left(\frac{\alpha \pi}{2}\right)-4 D_{3}^{2} r_{3}^{2} \cos ^{2}(\alpha \pi), \\
h_{7} & =2\left(-r_{3} r_{4}+q_{3} q_{4}\right) \cos \left(\frac{\alpha \pi}{2}\right)+2 q_{2} \cos \left(\frac{3 \alpha \pi}{2}\right)-8 r_{3} r_{4} D_{3}^{2} \cos \left(\frac{\alpha \pi}{2}\right) \cos (\alpha \pi), \\
h_{8} & =q_{4}^{2}-r_{4}^{2}+2 q_{3} q_{5} \cos (\alpha \pi)-4 r_{4}^{2} D_{3}^{2} \cos 2\left(\frac{\alpha \pi}{2}\right), \\
h_{9} & =2 q_{4} q_{5} \cos \left(\frac{\alpha \pi}{2}\right), \\
h_{10} & =q_{5}^{2}-D_{3}^{2} .
\end{aligned}
$$

\section{REFERENCES}

1. HIV/AIDS - World Health Organization report (2019).

2. Cai L., Li X., Ghosh M., Guo B. Stability analysis of an HIV/AIDS epidemic model with treatment. Journal of Computational and Applied Mathematics. 2009. V. 28. P. 313-323.

3. Hernandez-Vargas E.A.,Middleton R.H. Modeling the three stages in HIV infection. Journal of Theoretical Biology. 2013. V. 320. P. 33-40.

4. Swarnali S.S., Samanta G.P. Dynamical behavior of an HIV/AIDS epidemic model. Differential Equations and Dynamical Systems. 2014. V. 22. P. 369-395.

5. Simpson L., Gumel A. B. Mathematical assessment of the role of pre-exposure prophylaxis on HIV transmission dynamics. Applied Mathematics and Computation. 2017. V. 293. P. 168-193.

6. Akrami M.H., Atabaigi A. Hopf and forward bifurcation of an integer and fractional-order SIR epidemic model with logistic growth of the susceptible individuals. Journal of Applied Mathematics and Computing. 2020. V. 64. P. 615-633.

7. Wu L., Li Z., Zhang Y., Xie B. Complex Behavior Analysis of a Fractional-Order Land Dynamical Model with Holling-II Type Land Reclamation Rate on Time Delay. Discrete Dynamics in Nature and Society. 2020. V. 2020.

8. Shaikh A.S., Shaikh I.N., Nisar K.S. A mathematical model of COVID-19 using fractional derivative: outbreak in India with dynamics of transmission and control. Advances in Difference Equations. 2020. V. 373.

9. Wang Y., Liu K., Lou Y. An age-structured within-host HIV model with T-cell competition. Nonlinear Analysis: Real World Applications. 2017. V. 38. P. 1-20.

10. Xu J., Geng Y., Zhou Y. Global dynamics for an age-structured HIV virus infection model with cellular infection and antiretroviral therapy. Applied Mathematics and Computation. 2017. V. 22. P. 3721-3747.

11. Gakkhar S., Chavda N. A dynamical model for HIV-TB co-infection. Applied Mathematics and Computation. 2012. V. 218. P. 9261-9270.

12. Pinto C., Carvalho A. New findings on the dynamics of HIV and TB coinfection models. Applied Mathematics and Computation. 2014. V. 242. P. 36-46.

13. Krishnapriya P., Pitchaimani M., Witten T.M. Mathematical analysis of an influenza A epidemic model with discrete delay. Journal of computational and Applied Mathematics. 2017. V. 324. P. 155-172.

14. Monica C., Pitchaimani M. Analysis of stability and Hopf bifurcation for HIV-1 dynamics 
with PI and three intacelluar delays. Nonlinear Analysis:Real World Applications. 2016. V. 27. P. 55-69.

15. Krishnapriya P., Pitchaimani M. Analysis of time delay in viral infection model with immune impairment. Journal of Applied Mathematics and Computing. 2017. V. 27. P. 421-453.

16. Pitchaimani M., Monica C., Divya M. Stability analysis for HIV infection delay model with protease inhibitor. BioSystems. 2013. V. 114. P. 118-124.

17. Zafar Z.U.A., Ali N., Shah Z., Roy P., Zaman G., Deebani W. Hopf bifurcation and global dynamics of time delayed Dengue model, Computer Methods and Programs in Biomedicine. 2020. V. 195.

18. Zafar Z.U.A., Ali N., Zaman G., Thounthong P., Tunc C. Analysis and numerical simulations of fractional order Vallis system. Alexandria Engineering Journal. 2020. V. 59. P. 2591-2605.

19. Zafar Z.U.A. Fractional order Lengyel-Epstein chemical reaction model. Computational and Applied Mathematics. 2019. V. 38.

20. Zafar Z.U.A., Mushtaq M., Rehan K. A non-integer order dengue internal transmission model. Advances in Difference Equations. 2018. V. 23.

21. Zafar Z.U.A, Rehan K., Mushtaq M. HIV/AIDS epidemic fractional-order model. Journal of Difference Equations and Applications. 2017. V. 23. P. 1298-1315.

22. Zafar Z.U.A, Rehan K., Mushtaq M. Fractional-order scheme for bovine babesiosis disease and tick populations. Advances in Difference Equations. 2017. V. 86.

23. Kheiri H., Jafari M. Optimal control of a fractional-order model for the HIV/AIDS epidemic. International Journal of Biomathematics. 2018. V. 11.

24. Mondal S., Lahiri A., Bairagi N. Analysis of a fractional order eco-epidemiological model with prey infection and type 2 functional response. Mathematical Methods in the Applied Sciences. 2017. V. 40. P. 6776-6789.

25. Fatmawati, Shaiful E.M., Utoyo M.I. A Fractional-Order Model for HIV Dynamics in a Two-Sex Population. International Journal of Mathematics and Mathematical Sciences. 2018. V. 2018. P. 1-11.

26. Arafa A.A.M., Khalil M., Sayed A. A Non-Integer Variable Order Mathematical Model of Human Immunodeficiency Virus and Malaria Coinfection with Time Delay. Complexity. 2019. V. 2019. P. 1-13.

27. El-Sayed A.M., Arafa A.A., Khalil M., Sayed A. Backward bifurcation in a fractional order epidemiological model. Progress in Fractional Differentiation and Applications. 2017. V. 3. P. 281-287.

28. El-Sayed A.M., Arafa A.A., Khalil M., Sayed A. Mathematical Model of Vector-Borne Plant Disease with Memory on the Host and the Vector. Progress in Fractional Differentiation and Applications. 2016. V. 2. P. 277-285.

29. Arafa A.A.M., Rida S.Z., Khalil M. The effect of anti-viral drug treatment of human immunodeficiency virus type 1 (HIV-1) described by a fractional order model. Applied Mathematical Modelling. 2013. V. 37. P. 2189-2196.

30. Arafa A.A.M., Rida S.Z, Khalil M. Fractional modeling dynamics of HIV and CD4+ T-cells during primary infection. Nonlinear Biomedical Physics. 2012. V. 6. P. 1-7.

31. Arafa A.A.M., Rida S.Z., Khalil M. A fractional-order model of HIV infection: numerical solution and comparisons with data of patients. International Journal of Biomathematics. 2014. V. 7.

32. Das M., Samanta G. Stability analysis of a fractional ordered COVID-19 model. Computational and Mathematical Biophysics. 2021. V. 9. P. 22-45.

33. Das M., Samanta G. A delayed fractional order food chain model with fear effect and prey refuge. Mathematics and Computers in Simulation. 2020. V. 178. P. 218-245. 
34. Yan Y., Kou C. Stability analysis for a fractional differential model of HIV infection of $C D 4^{+}$T-Cells with time delay. Mathematics and Computers in Simulation. 2012. V. 82. P. $1572-1585$.

35. Wang X., Wang Z. Dynamic Analysis of a Delayed Fractional-Order SIR Model with Saturated Incidence and Treatment Functions. International Journal of Bifurcation and Chaos. 2018. V. 28.

36. Rihan F.A., Al-Mdallal Q.M., AlSakaji H.J., Hashish A. A fractional-order epidemic model with time-delay and nonlinear incidence rate, Chaos, Solitons and Fractals, 126, (2019) 97-105.

37. Rihan F.A., Lakshmanan S., Hashish A. H., Rakkiyappan R., Ahmed E. Fractional-order delayed predator-prey systems with Holling type-II functional response. Nonlinear Dynamics. 2015. V. 50. P. 777-789.

38. Carvalho A., Pinto C.M.A. A delay fractional order model for the co-infection of malaria and HIV/AIDS. International Journal of Dynamics and Control . 2017. V. 5. P. 168-186.

39. Chinnathambi R., Rihan F.A., Alsakaji H.J. A fractional-order model with time delay for tuberculosis with endogenous reactivation and exogenous reinfections. Mathematical Methods in the Applied Sciences. 2019. P. 1-15.

40. Sontakke B.R., Shaikh A.S. Properties of Caputo Operator and Its Applications to Linear Fractional Differential Equations. International Journal of Engineering Research and Applications. 2015. V. 5. P. 22-27.

41. Diekmann O., Heesterbeek J.A.P., Metz J.A.J. On the definition and the computation of the basic reproduction ratio $R_{0}$ in models for infectious diseases in heterogeneous populations. Journal of Mathematical Biology. 1990. V. 28. P. 365-382.

42. Marino S., Hogue I.B., Ray C.J., Kirschner D.E. A methodology for performing global uncertainty and sensitivity analysis in systems biology. Journal of Theoretical Biology. 2008. V. 254. P. 178-196.

43. Bhalekar S., Daftardar-Gejji V. A Predictor-Corrector Scheme for Solving Nonlinear Delay Differential Equations of Fractional Order. Journal of Fractional Calculus and Applications. 2011. V. 1. P. 1-9.

44. Naik P.A. Global dynamics of a fractional-order SIR epidemic model with memory. International Journal of Biomathematics. 2020. V. 13.

45. WHO. Global Health Observatory data repository. HIV/AIDS. URL: https://apps.who.int/gho/data/view.main; 2020. Retrieved : 2020-07-09.

Accepted 12 December 2020.

Revised 20 April 2021.

Published 12 May 2021. 